\title{
The Effects of Spectral Smearing and Elevated Thresholds on Speech in Noise Recognition in Simulated Electric-Acoustic Hearing
}

\author{
Aretha Mulder
}

A thesis submitted in partial fulfilment of the requirements for the Degree of Master of Audiology at the University of Canterbury

February 2014 


\section{Table of Contents}

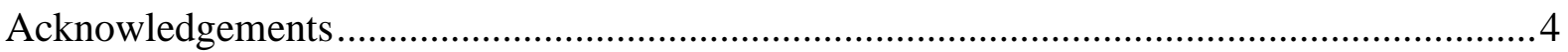

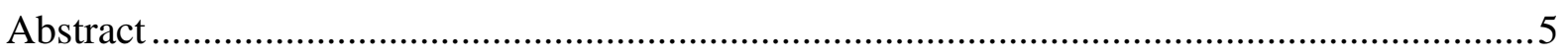

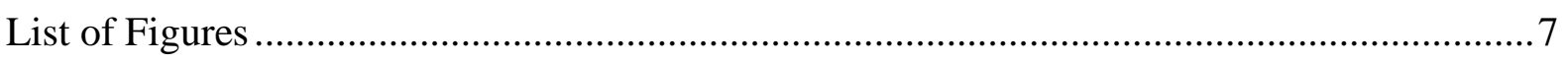

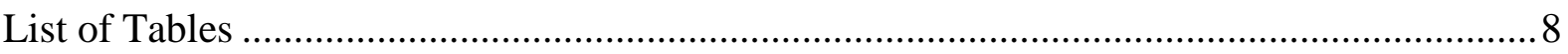

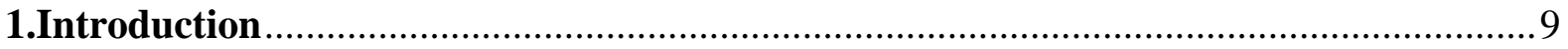

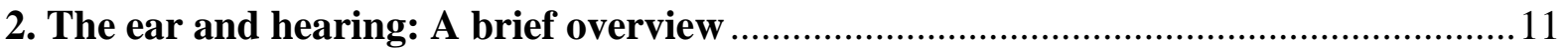

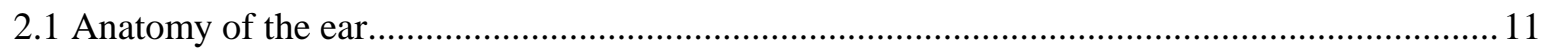

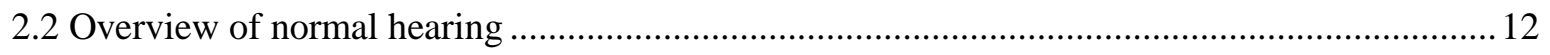

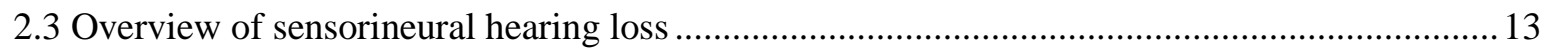

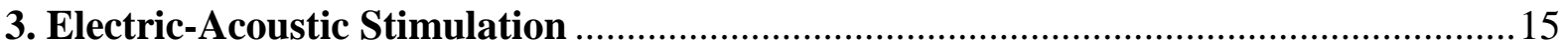

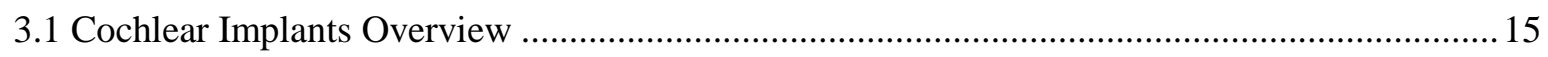

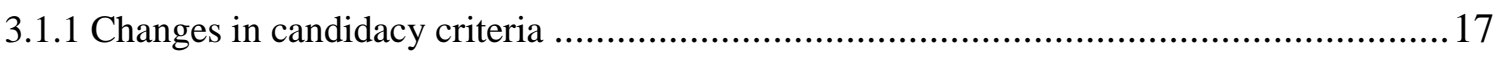

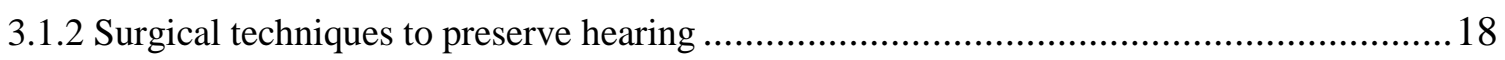

3.1.3 Overview of a method used to simulate cochlear implant processing ................................20

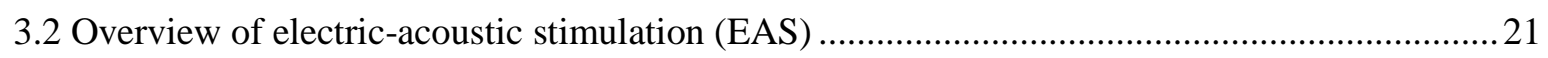

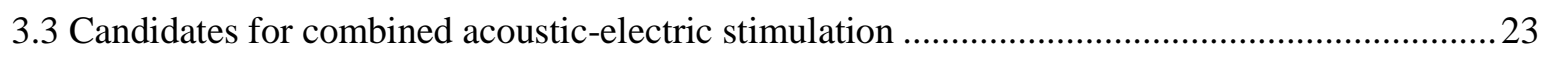

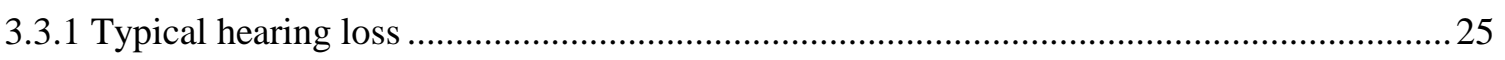

4. Benefits of combined EAS: A review of the literature...............................................2 27

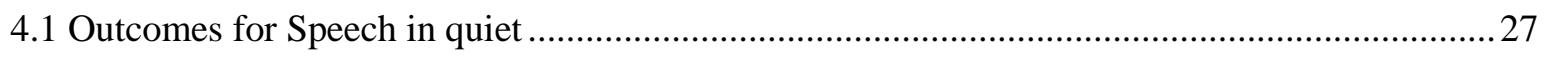

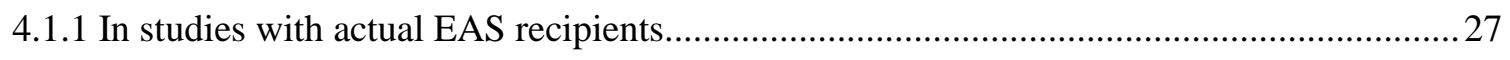

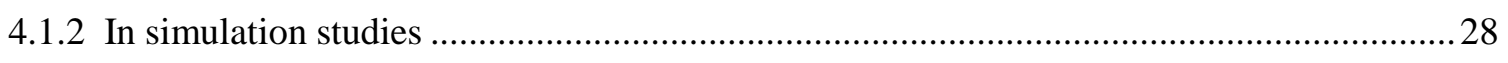

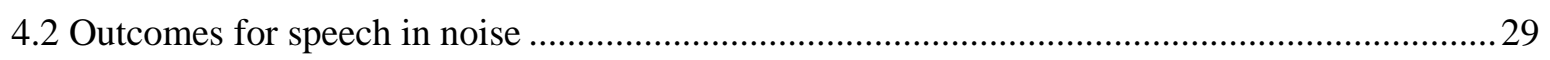

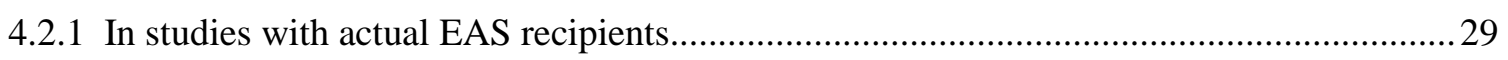

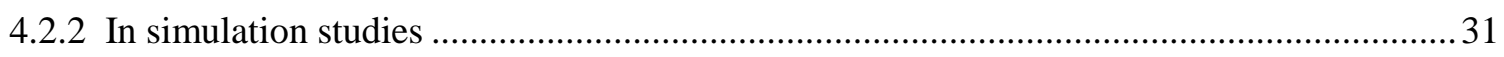

4.3 Outcomes for Melody Recognition and Subjective sound quality........................................... 33

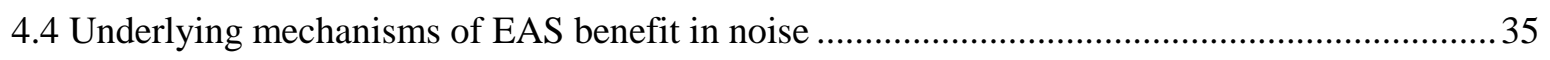

4.5 Comparison of outcomes between simulation studies and studies with actual EAS recipients .. 40

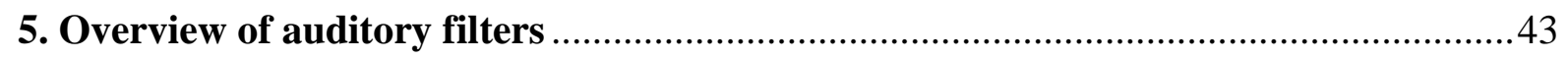

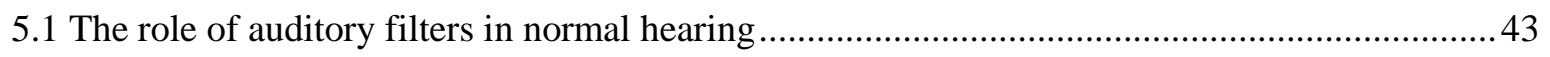

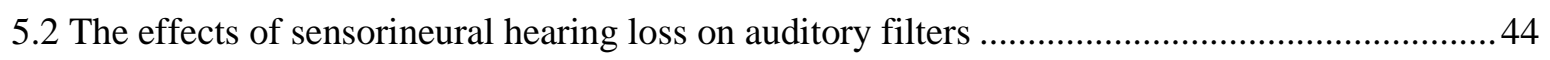

5.3 Overview of a method used to simulate broadened auditory filters........................................... 46 


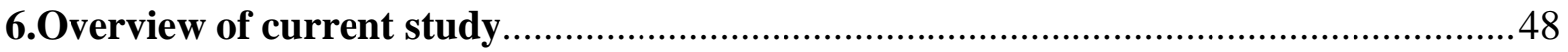

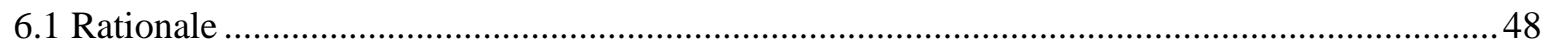

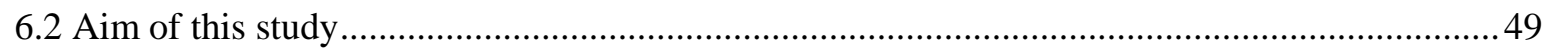

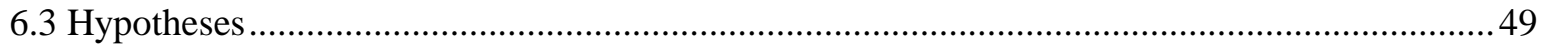

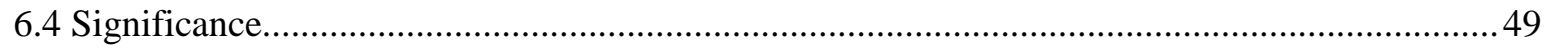

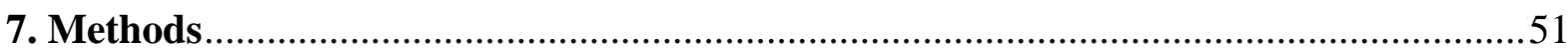

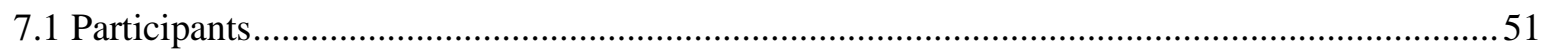

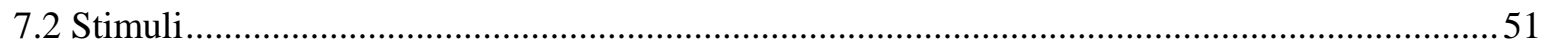

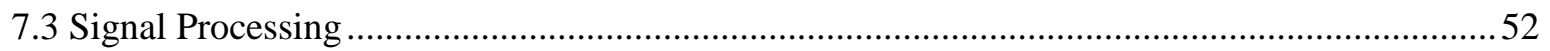

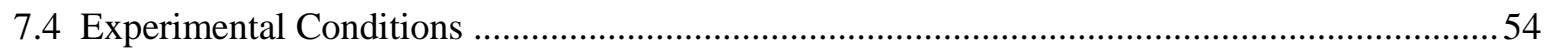

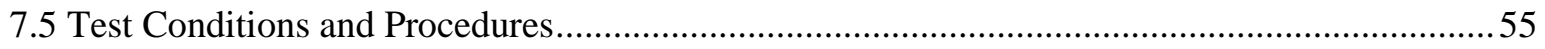

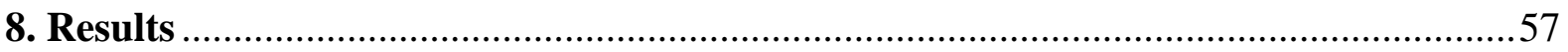

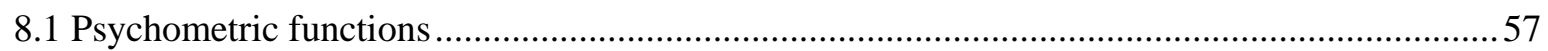

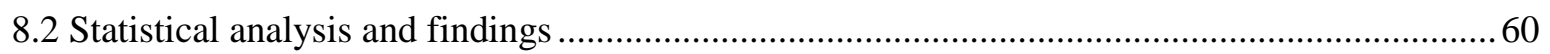

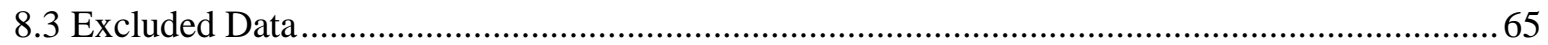

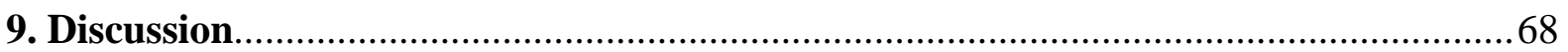

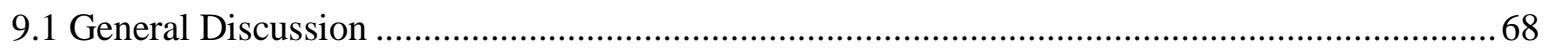

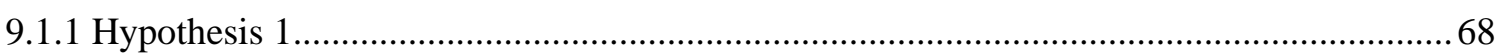

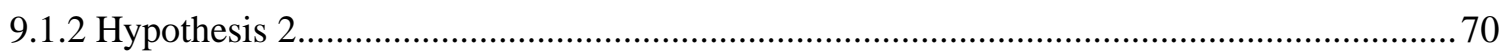

9.2 Comparisons of EAS benefit with previous literature ….............................................................. 74

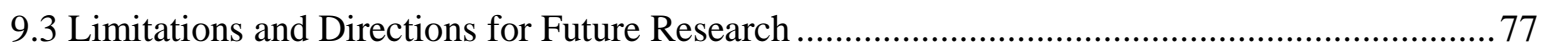

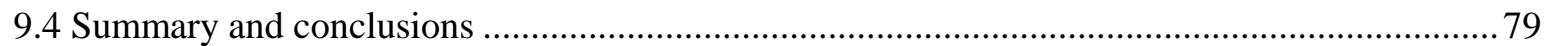

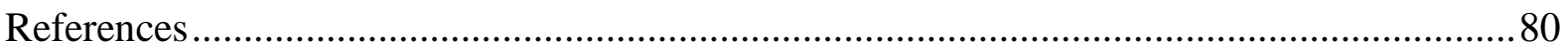

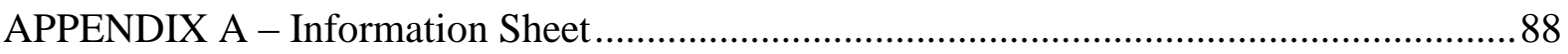

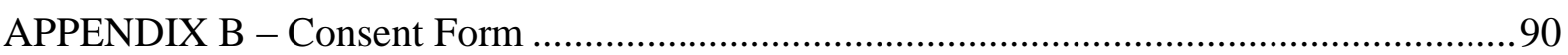




\section{Acknowledgements}

First I would like to thank my senior supervisor, Dr. Donal Sinex, without whom I would not have been able to conduct this thesis in my field of interest. His knowledge and experience was invaluable and his patience, time and effort much appreciated. Thank you for maintaining such a positive attitude throughout this process and for consistently pointing me in the right direction.

I would also like to thank my assistant supervisor, Dr. Emily Lin, who was always willing to help and whose expertise in statistics proved indispensable. Likewise I am grateful to Anne O'Connell for her efficiency in helping me organize incentives, equipment, and other necessary errands. I would like to extent a general thank you to all the Communication Disorders staff, be it academic, clinical, or administrative. As someone who has been a student in this department for six years, I know what a dedicated, helpful, and approachable group of people you are.

I would like to express my gratitude to all those who participated in this study. Data collection would not have been possible without the commitment in both time and energy from you.

Furthermore, I would like to thank my classmates for their friendship and reassurance. No one understood what I was going through better than you. You are all a source of inspiration and make me endlessly proud of the profession I am about to enter.

Last but not least I would like to thank my wonderful friends, family, and my partner Joshua. Your love, support and encouragement is my motivation for all I have achieved and still will achieve in life. 


\begin{abstract}
Combined Electric-acoustic stimulation (EAS) is becoming an increasingly viable treatment option for individuals with sloping severe to profound high frequency hearing loss and residual low frequency hearing. Sound stimulation via EAS is delivered to the high frequency region electrically using cochlear implantation, and to the low frequency region acoustically with or without amplification from hearing aids. This combined mode of stimulation often results in improved speech recognition in background noise compared to either mode of stimulation in isolation. It is important to note that many EAS listeners have some degree of hearing loss in the low frequency region, and may experience associated effects such as reduced frequency selectivity and elevated audiometric thresholds. This study simulated EAS listening in 20 normal hearing listeners by combining vocoded high frequency sound with low frequency sound. Low frequency sound was further manipulated by applying varying degrees of spectral smearing and attenuation to the low frequency region in an EAS simulation, to simulate changes in frequency selectivity and sensitivity that usually accompany sensorineural hearing loss. The aim of this study was to investigate the effects of spectral smearing and attenuation of low frequency information on the identification of vocoded speech in noise. Participants were required to complete a sentence recognition task in the presence of competing talkers for six simulated listening conditions with varying degrees of processing in the low frequency region. Results indicated that the advantage for speech in noise of simulated combined EAS over simulated electric stimulation alone was 3.9 $\mathrm{dB}$ when low frequency sound was unprocessed, $2.9 \mathrm{~dB}$ when low frequency sound had spectral smearing of $\mathrm{x} 3$ applied, and $2.4 \mathrm{~dB}$ when low frequency sound had spectral smearing of $\mathrm{x} 6$ applied. When $30 \mathrm{~dB}$ attenuation was applied as well as $\mathrm{x} 3$ spectral smearing, no significant benefit was observed. When $60 \mathrm{~dB}$ attenuation was applied as well as $\mathrm{x} 3$ spectral
\end{abstract}


smearing, a significant negative relationship was found, with a $3 \mathrm{~dB}$ disadvantage found for simulated EAS compared to simulated electric stimulation alone. Overall, the results of this study indicate that there is indeed a significant improvement in speech recognition in a background of competing speakers with simulated EAS compared to simulated electric stimulation only. However, when reduced hearing thresholds were simulated for the residual low frequency hearing, we found that this benefit was either absent or reversed. These results therefore support the use of amplification for individuals with reduced hearing thresholds in the low frequencies in order to utilize the benefit they are able to achieve with combined EAS. 


\section{List of Figures}

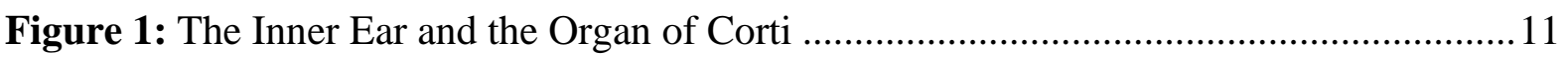

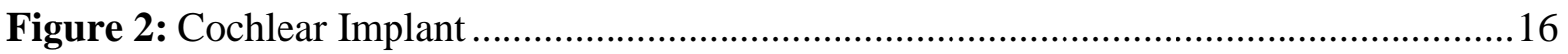

Figure 3: Candidacy criteria for Hybrid cochlear implantation......................................... 24

Figure 4: Pre-operative hearing thresholds of EAS candidates .........................................26

Figure 5: Derived auditory filter shapes at $1 \mathrm{kHz}$ in normal and impaired ears...................45

Figure 6: Spectral smearing applied to a single vowel isolated from a sentence in quiet, as applied in the current study.....

Figure 7: Psychometric function of speech recognition score as a function of SNR for

Participant 1 .58

Figure 8: Psychometric function of speech recognition score as a function of SNR for

Participant 16

Figure 9: Psychometric function of speech recognition score as a function of SNR for

Participant 18

Figure 10: Box Plot of SRT for each condition.

Figure 11: Benefit (dB) of EAS listening conditions compared to VC condition

Figure 12: SRT (dB SNR) of the VC condition and three EAS conditions with no attenuation applied to the low frequency region.

Figure 13: SRT (dB SNR) of EAS conditions with $x 3$ spectral smearing and varying degrees of attenuation applied to the low frequency region .64

Figure 14: Participant 5 PRE- and POST psychometric functions illustrating speech perception scores as a function of $\mathrm{dB}$ SNR at the start of the experiment and at the end of the experiment for Condition 6 . .66

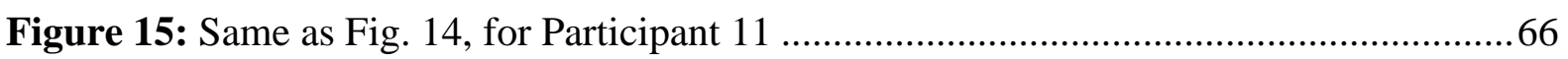

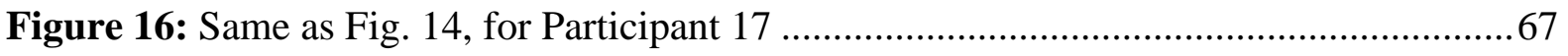




\section{List of Tables}

Table 1: Cut-off frequencies for cochlear implant simulation (Arehart et al., 2011) .52

Table 2: Individual SRTs for each condition 64

Table 3: Comparing EAS benefit between a group of clinical trials and the current study.... 76 


\section{Introduction}

Combined electric-acoustic stimulation (EAS) via Hybrid cochlear implants is a relatively new and controversial treatment for severe-profound sloping high frequency hearing loss. A hybrid cochlear implant is a device which is able to simultaneously deliver sound both acoustically, with or without additional amplification, and electrically, via cochlear implant technology. This has recently been considered an option for those individuals who are unable to achieve satisfactory speech recognition with traditional amplification despite residual hearing in the low frequencies. There has been evidence to suggest that for these individuals, combined EAS provides significant benefit for speech in noise recognition compared to electric only or acoustic only stimulation (Gantz, Turner, Gfeller, \& Lowder, 2005; Gantz, Turner, \& Gfeller, 2006; Kiefer et al., 2005; Lorens, Polak, Piotrowska, \& Skarzynski, 2008; Turner, Gantz, Vidal, Behrens, \& Henry, 2004; Turner, Gantz, \& Reiss, 2008).

Residual low frequency hearing is a vital component of EAS. Residual hearing refers to any hearing that is 'left over' following hearing loss. One cannot assume that residual hearing will be normal. Any degree of hearing that allows for acoustic sound perception can be labelled residual hearing and therefore contributes to EAS. This means that residual hearing can fall anywhere in the range from normal hearing, to profound hearing loss. The aim of this study was to determine to what extent the benefit of residual low frequency hearing for recognition of speech in noise in simulated EAS hearing is affected by reduced frequency selectivity and increased audiometric thresholds. This was achieved by applying vocoding to the high frequency range and various degrees of spectral smearing and attenuation to the low frequency range in normal hearing listeners, in order to simulate electric-acoustic hearing. 
It is believed that the current research will add to the understanding of a clinicallyimportant problem: The degree of benefit Hybrid cochlear implant recipients might expect based on their residual hearing status. An attempt has been made to address this question through clinical trials and studies in which real EAS recipients were recruited. However, caution has to be taken when generalizing results as a large number of extraneous variables, such as age at implantation and duration of hearing loss, are likely to affect an individual's performance outcomes following Hybrid cochlear implant surgery. Furthermore, this question has not been addressed by simulation studies to date. 


\section{The ear and hearing: A brief overview}

\subsection{Anatomy of the ear}

The human ear consists of the outer ear, the middle ear, and the inner ear. The primary function of the outer ear is to act as an antenna and to assist with sound localisation. The middle ear functions as an impedance matcher to transfer the air-borne sound waves to the fluid filled inner ear. The inner ear consists of the cochlea and the vestibular system. The cochlea is the spiral shaped peripheral hearing organ. Inside the cochlea there are three parallel spaces known as the scala vestibuli, the scala media, and the scala tympani. The scala media is separated from the scala vestibuli via Reissner's membrane, and from scala tympani via the basilar membrane. Along the medial edge of scala media the organ of Corti can be found. The organ of Corti contains the cochlear receptor cells: inner hair cells and outer hair cells. These synapse with the efferent and afferent auditory nerve fibres which send nerve impulses via the brainstem to the brain. Protruding from the outer and inner hair cells are stereocilia. Outer hair cell steriocillia are embedded in the gelatinous tectorial membrane.

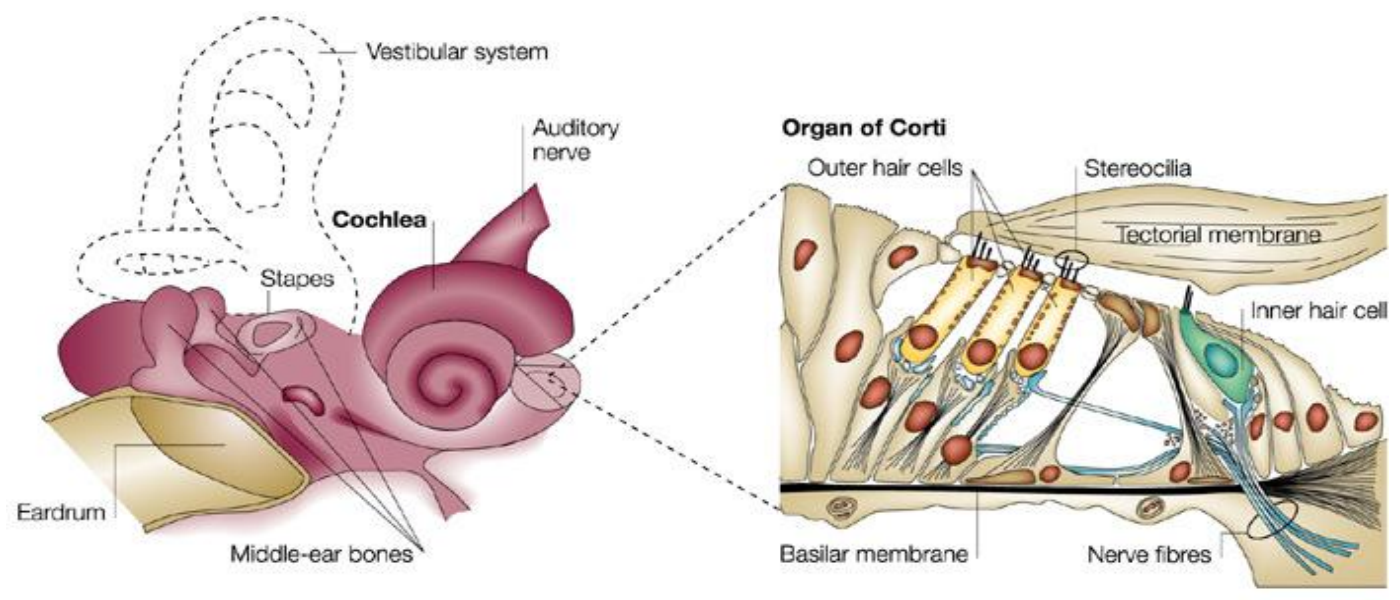

Nature Reviews | Molecular Cell Biology

Figure 1: The Inner Ear and the Organ of Corti

Adapted by permission from Macmillan Publishers Ltd: Nature Reviews Molecular Cell Biology 3, 104-111 (February 2002), doi:10.1038/nrm730 


\subsection{Overview of normal hearing}

Sound waves enter the external ear and, through a series of impedance matching mechanisms, are transferred through the middle ear into the inner ear fluids. In the inner ear these sound waves result in longitudinal vibration of the basilar membrane. At the base of the cochlea the basilar membrane is stiff and narrow, while at the apex it becomes broader and more flexible. It is these mechanical properties of the basilar membrane that lend the cochlea its tonotopicity, as different frequencies result in maximal vibration along different places of the basilar membrane. High frequencies cause maximal vibration at the basal region and low frequencies cause maximal vibration at the apical region. The pattern of basilar membrane vibration is known as a travelling wave which grows in magnitude as it travels to its point of maximal vibration after which the motion trails off rather abruptly (von Békésy, 1960).

The mechanical properties of the basilar membrane, however, are not enough to explain the magnitude of displacement and the resulting 'fine tuning' at the characteristic frequency of the incoming signal. This can be attributed to the active process of the outer hair cells. As the basilar membrane moves up and down in response to sound, a shearing motion exists with the tectorial membrane which deflects the stereocilia of the outer hair cells. This excitation of outer hair cells causes them to change shape and increase basilar membrane displacement. The fluid movement within the organ of Corti as the basilar membrane is set in motion will also result in deflection of the stereocillia of the inner hair cells. If the magnitude of this motion is sufficient it will result in depolarization of the hair cells and release of neurotransmitter which ultimately results in excitation of the cochlear nerve cells. This excitation of nerve cells travels along the brainstem to the auditory cortex in the brain. The brainstem and auditory cortex processes the incoming signal, which ultimately results in the perception of sound. 


\subsection{Overview of sensorineural hearing loss}

Hearing loss can be classified by its type, its degree, and its configuration. The two main types of hearing loss are conductive hearing loss and sensorineural hearing loss. Conductive hearing loss occurs as a result of dysfunction in the outer and/or middle ear which prevents sound from reaching the inner ear with adequate intensity. Sensorineural hearing loss occurs when there is damage to the inner ear, or the pathways from the inner ear to the brain. One of the most common causes of sensorineural hearing loss is absent or dysfunctional outer hair cells. This results in a loss of the active process by which outer hair cells increase basilar membrane vibration for low level sounds, thus 'fine-tuning' the response to sound at that frequency of stimulation (von Békésy, 1960). Hearing loss due to outer hair cell loss/damage typically results in a moderate to moderately-severe degree of hearing loss. In addition to reduced thresholds this type of hearing loss is also likely to result in broadened 'tuning' of the frequency response and therefore reduced frequency selectivity (Turner, 2006). Hearing loss of this type is typically treated using traditional amplification such as hearing aids.

Sensorineural hearing loss may also involve loss/damage of the inner hair cells. Considering inner hair cells are important for the detection of a sound, this often results in a more severe to profound degree of hearing loss. When the inner hair cell damage is extensive enough we might even refer to the place of damage as a 'dead region' (Vickers, Moore, \& Baer, 2001). This type of hearing loss is typically not able to be treated with traditional amplification, and cochlear implantation, which bypasses the inner hair cells, might be indicated (Turner, 2006). The relevant hearing loss configuration to the current study can be described as 'sloping' or 'steeply sloping'. This hearing loss configuration is applicable when hearing in the low-mid frequencies are better than hearing in the mid-high frequencies. 
One of the methods used to simulate the effects of hearing loss is to attenuate the overall sound level of the signal in order to simulate reduced hearing thresholds. Methods used to simulate additional consequences of hearing loss will be described at a later stage in this review. 


\section{Electric-Acoustic Stimulation}

\subsection{Cochlear Implants Overview}

A cochlear implant is a device which aims to restore the perception of sound in individuals with profound hearing loss. This is achieved by direct stimulation of the auditory nerve through electrical currents, thus bypassing damaged or absent cochlear hair cells. The cochlear implant consists of a surgically implanted internal component with electrodes placed inside the cochlea, and an external component responsible for picking up and processing sounds from the environment. The external component consists of a battery compartment, a microphone, a speech processor, and a transmitting coil. The internal component consists of the receiver stimulator and the electrode array. The microphone picks up external sounds, which are then encoded by the speech processor and converted into an electrical signal which is transferred across the skin by electromagnetic induction to the internal receiver stimulator. The receiver stimulator converts this signal into electrical pulses which are transmitted via the electrode array to the cochlear nerve (Wilson, 2004). 


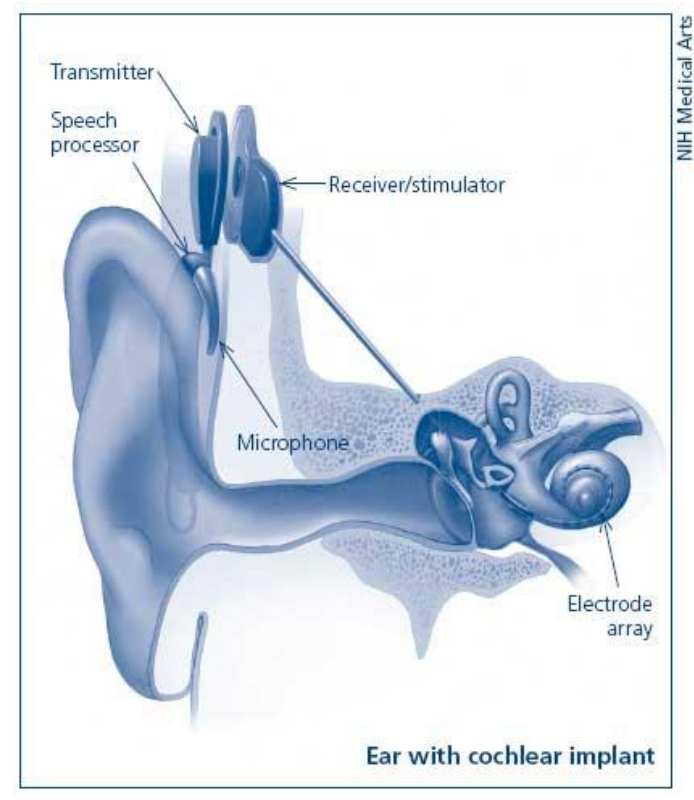

Figure 2: Cochlear Implant

Figure obtained from http://www.nidcd.nih.gov/health/hearing/pages/coch.aspx. Credit to National Institutes of Health Image Bank/Department of Health and Human Services

The typical processing strategies for cochlear implants involve band-pass filtering the speech into different frequency bands, extracting the amplitude envelope within each band, and using the amplitude envelope to modulate the electrical pulses delivered by the electrodes to the cochlear nerve (Wilson et al., 1991). This means that cochlear implant recipients rely on temporal envelope modulations for pitch perception. This processing strategy removes both spectral and temporal fine structure cues, which results in reduced pitch perception (Shannon, Fu, Galvin, \& Friesen, 2004). Loudness perception with cochlear implants is dependent on the amount of electrical current that can be passed through the electrode that will not be painful, but will be detectable. These factors limit the loudness and pitch perception of cochlear implant recipients as compared to that of normal hearing individuals. This reduction in pitch perception has shown to have a detrimental effect on speech recognition, particularly in the presence of background noise (Cullington \& Zeng, 2008) 
Cochlear implant surgery takes approximately 2-3 hours under general anaesthesia. First an incision is made in the skin behind the ear to expose the mastoid and temporal bone. A depression/pocket is then drilled into the bone for placement of the receiver stimulator. Once the middle ear cavity is exposed and the oval and round windows identified, a cochleostomy (2-3mm hole) is drilled through the wall of the cochlea. The electrode array can then be inserted into the cochlea (Clark, Franz, Pyman, \& Webb, 1991). Traditionally, the goal during electrode array insertion was to get the electrode array as close to the apex of the cochlea as possible. This was in order to achieve a maximum longitudinal distance of stimulation of the basilar membrane and therefore as large a range of frequency stimulation as possible. At the conclusion of the surgery the areas surrounding the cochlea are packed with soft tissue, the cochleostomy is closed, and the incision is closed using sutures. Approximately a month post surgery the cochlear implant is switched on and mapped. Cochlear implant mapping refers to the process of determining the minimum level of electrical stimulation required to detect a sound, and the maximum level of electrical stimulation that is not uncomfortable, for each electrode (Clark, 2003).

Even though greatly improved speech recognition and environmental sound awareness has been reported with cochlear implants, it remains an invasive procedure and more importantly one that involves extensive rehabilitation and patient motivation. Furthermore, outcomes are highly variable amongst individuals and it is difficult if not impossible to predict who would do well with a cochlear implant and who won't (American Speech-Language-Hearing Association, 2004).

\subsubsection{Changes in candidacy criteria}

Since the approval of a single channel cochlear implant by the United States Food and Drug Administration (FDA) in 1984, significant progress has been made in cochlear implant 
technology and candidacy criteria (Sampaio, Araújo, \& Oliveira, 2011; Wilson, Lawson, Müller, Tyler, \& Kiefer, 2003). One of the major advances in candidacy criteria is that individuals with increasing levels of residual hearing are being considered for cochlear implantation (Sampaio et al., 2011; Wilson et al., 2003). This expansion of candidacy criteria has gone hand in hand with the improved performance outcomes achieved by cochlear implant recipients. Speech understanding for many cochlear implant patients now exceed that of individuals with severe to profound hearing loss fit with traditional acoustic amplification (Kiefer et al. 2005). Another driving force for this change is the refinement of surgical techniques and electrode array design that makes preservation of residual acoustic hearing a realistic goal for many cochlear implant recipients.

\subsubsection{Surgical techniques to preserve hearing}

A number of studies have shown how residual hearing may be preserved. Gantz et al. (2006) reported preliminary results of the United States Food and Drug Administration (FDA) clinical trial of the Iowa/Nucleus Hybrid 10-mm cochlear implant. In this clinical trial a short electrode array $(10 \mathrm{~mm})$ was implanted using surgical techniques similar to those used for a standard length $(27 \mathrm{~mm})$ electrode array, with a few notable differences: low speed drilling to prevent acoustic trauma to the cochlea, no suctioning of the perilymph, and slow and careful advancement and positioning of the electrode with particular care given to the location of insertion. The goal of short electrode insertion is to electrically stimulate those areas of the cochlea which have lost cochlear hair cells and acoustic sound perception, while taking care not to insert the electrode deep enough to cause damage to the areas of the cochlea that still have surviving cochlear hair cells and residual hearing. The electrode is therefore inserted basaly to stimulate the high frequency region, while not protruding into the apical low frequency region. Gantz et al. (2006) described the use of a guard on the electrode array which prevents the electrode array from penetrating too deep into the cochlea. 
Forty-eight participants with residual low frequency hearing (thresholds $<60 \mathrm{~dB}$ at $500 \mathrm{~Hz}$ and below) were implanted with the Iowa/Nucleus Hybrid cochlear implant. Immediately following surgery, $47 / 48$ of the patients in this clinical trial had preserved hearing. One patient lost hearing after 2.5 months following a viral infection and three further patients experienced a threshold decrease of $30 \mathrm{~dB}$ or more 2-3 months following surgery. Further clinical trials of the Iowa/Nucleus Hybrid cochlear implant resulted in threshold shifts of $20 \mathrm{~dB}$ or less for the majority (70\%) of patients (Turner et al., 2008).

Kiefer et al. (2004) approached hearing preservation by identifying a list of factors that can contribute to immediate or eventual loss of hearing and developed their surgical techniques to address these issues. The following factors were identified: acoustic trauma as a result of high speed drilling, physical and mechanical damage to cochlear structures during electrode insertion, cochlear fluid homeostasis disturbance, bacterial infection, cochlear fibrosis. This approach allowed them to achieve hearing preservation to within $20 \mathrm{~dB}$ of preoperative thresholds in $12 / 14$ patients and hearing preservation to within $10 \mathrm{~dB}$ of preoperative thresholds in 9/14 patients. A later study by Gstoettner et al. (2009) revealed complete hearing preservation in 4/9 patients, and partial hearing preservation in 5/9 patients. These results indicated that all patients in this study were able to achieve some level of hearing preservation.

To summarize, although by no means guaranteed, some degree of preservation of residual hearing seems to be a highly achievable goal for cochlear implant recipients. It is important to note that hearing preservation was partial for the majority of patients. This highlights the fact that the majority of hybrid cochlear implant recipients will present with some hearing loss in the low frequencies. The predominant methods used to assist with the preservation of residual hearing include careful surgical techniques; newer, smaller, and softer electrode arrays; protecting the inner ear through methods such as hypothermia, 
corticosteroids, antioxidants and anti-apoptosis agents; and continuous monitoring of hearing during surgery (von Ilberg, Baumann, Kiefer, Tillein, \& Adunka, 2011).

\subsubsection{Overview of a method used to simulate cochlear implant processing}

A vocoder is a system which analyzes and synthesizes speech into an electronically transferable format by compressing it into multiple frequency bands. Speech vocoding was first developed and described by Dudley (1939) and was originally intended for telecommunications use. Noise-vocoded speech is created by dividing the speech signal into frequency bands, extracting the amplitude envelope from each frequency band, modulating noise in each frequency band with the extracted amplitude envelope, and recombining the frequency bands to produce the noise-vocoded speech signal.

Shannon, Zeng, Kamath, Wygonski, and Ekelid (1995) were amongst the first to describe the use of this method of speech processing to simulate the stimulation pattern of a cochlear implant. They achieved this by preserving the amplitude and temporal cues of a speech signal while varying the amount of spectral information (number of noise bands) which can be said to simulate varying numbers of channels in cochlear implant processing. Not surprisingly, they found that speech recognition improved as the number of bands/channels increased. What was surprising however, was that good speech recognition scores $(90 \%)$ in quiet were obtained with only three bands/channels. This indicates that sufficient speech recognition in quiet can be achieved even with very limited spectral information. Fishman, Shannon, and Slatery (1998) used these data from Shannon et al. (1995) as a comparison to their own data with real cochlear implant recipients. They found that the speech vocoder method used by Shannon et al. (1995) offered an approximation of the maximum performance of cochlear implant recipients, particularly for smaller numbers of bands/channels. 
Friesen, Shannon, Baskent, and Wang (2001) measured speech recognition as a function of the number of spectral channels and the signal to noise ratio for cochlear implant recipients and normal hearing listeners listening to vocoded speech. They found that for vowel, word, and sentence recognition, the best cochlear implant listeners performed similarly to the normal hearing simulation group, but only up to eight channels. For increasing numbers of channels, the cochlear implant group's performance remained relatively stable, while the normal hearing simulation group continued to show improved speech recognition up to 20 channels. One possible conclusion that can be drawn from these results is that simulation studies that simulate smaller numbers of channels (eight or less) are more likely to offer an approximation of performance for the highest performing cochlear implant users, compared to simulation studies that simulate larger numbers of channels.

The use of vocoding to simulate cochlear implant listening is widely reported in the literature (Dorman, Loizou, \& Rainey, 1997; Friesen et al., 2001; Li \& Loizou, 2009; Qin \& Oxenham, 2005; Shannon et al., 1995; Whitmall, Poissant, Freyman, \& Helfer, 2007). This method of simulation allows for manipulation of a number of cochlear implant processing parameters so they may be studied in isolation. These manipulations would not be possible in actual cochlear implant recipients, hence the popularity of simulation studies. One of the most obvious of these parameters is the number of bands/channels and its effect on various outcome measures. Other vocoding manipulations include the frequency ranges/cut-offs of simulated channels, the bandwidth of amplitude modulation, and the carrier type, with either noise or tones being used to modulate the amplitude envelope of the speech signal.

\subsection{Overview of electric-acoustic stimulation (EAS)}

EAS refers to simultaneous stimulation both electrically and acoustically to result in sound perception. The electrical stimulation is achieved via a cochlear implant directly 
stimulating the auditory nerve. The acoustic stimulation occurs due to stimulation of surviving cochlear hair cells.

The idea of combining electric and acoustic stimulation was first introduced by von Ilberg et al. (1999), who obtained promising results from both animal experiments and the clinical experience of a patient with partial hearing preservation following cochlear implant surgery. In order to test the feasibility of combined electric-acoustic stimulation, they argued three questions would need to be answered: (1) "Does simultaneous EAS interfere with the physiological discharge pattern of the auditory system?", (2) "Is a chronic electric stimulation hazardous to residual hair cells?", (3) "Is a simultaneous EAS beneficial to patients with severe high-frequency hearing loss?” (von Ilberg et al., 2009, p 335). Experimental data from cats and guinea pigs revealed that the answer to the first two questions is 'no'. Acute and chronic electric stimulation did not significantly interfere with acoustic transmission of sound via the auditory nerve, indicating these modalities can be used in conjunction. Their third question was addressed through clinical observations of a patient with partial hearing preservation following cochlear implantation. A range of speech tests were performed with the hearing aid alone, the cochlear implant alone, and a combination of both the hearing aid and the cochlear implant monaurally. Test scores were consistently highest for the simultaneous EAS (hearing aid + cochlear implant) listening condition. The patient also reported this listening condition to sound most pleasant subjectively. The authors concluded that combining electrical and acoustic stimulation did not produce detrimental interference in the auditory system, but rather that the central auditory system appears able to combine information from both modalities in an additive manner. Although this study presented the clinical data of only one patient, it nonetheless represents an important step towards the investigation and development of combined electric and acoustic stimulation, which was relatively unexplored prior to this time. 
Since then, clinical results from a large number of studies have indicated improved speech recognition for individuals receiving both electric and acoustic stimulation compared to either electric or acoustic stimulation alone (Gantz et al., 2005; Gantz et al., 2006; Gstoettner et al., 2009; Kiefer et al., 2005; Lorens et al., 2008; Turner et al., 2004; Turner et al., 2008). Although evident for speech in quiet, this benefit provided by EAS is most pronounced for speech in noise. Kiefer et al. (2005) demonstrated benefits of more than $70 \%$ in EAS conditions compared to cochlear implant alone conditions for speech in noise in individual patients. This effect in which scores under EAS conditions are greater than the simple addition of scores for electric alone and acoustic alone stimulation is commonly referred to as a synergistic effect (von Ilberg et al., 2011; Wilson et al., 2003).

\subsection{Candidates for combined acoustic-electric stimulation}

EAS is a viable option particularly for those individuals with severe-profound high frequency hearing loss, but with residual low frequency hearing. This is because the cochlear implant may be used to stimulate the basal end of the cochlea to achieve high frequency sound perception, while the intact apical end of the cochlea can be acoustically stimulated with or without amplification to achieve low frequency sound perception.

Traditionally individuals with sloping severe to profound high frequency hearing loss and residual low frequency hearing have been fit with hearing aids in an attempt to restore some of their speech perception. Although hearing aids are used successfully by many, a significant proportion of these individuals are unable to obtain benefit from traditional acoustic amplification (Vickers et al., 2001). They can 'hear' speech, but have insufficient clarity for intelligibility, something which a hearing aid is unable to amend. Even with rather well preserved low-frequency hearing (thresholds of 20-60 dB HL up to $1 \mathrm{kHz}$ ), the monosyllabic word scores of patients in a clinical study by Kiefer et al. (2004) generally 
remained below 40\% despite acoustic amplification. Kiefer et al. (2005) used monosyllabic word understanding of less than $40 \%$ in the best aided condition as one of their inclusion criteria for an EAS clinical trial.

Furthermore, these individuals have traditionally not been considered appropriate candidates for cochlear implantation due to the risk of loss of residual hearing. Fortunately, as outlined previously in this chapter, this risk has significantly reduced which has lead to the expansion of candidacy criteria. Figure 3 demonstrates the current candidacy criteria for preoperative hearing thresholds for Hybrid cochlear implantation. As seen in this figure, thresholds for low frequency hearing $(<750 \mathrm{~Hz})$ can range from normal to moderately-severe in degree, while thresholds for high frequency hearing $(>3 \mathrm{kHz})$ can range from severe to profound in degree. The sloping/steeply sloping hearing loss configuration of EAS candidates is also illustrated in this Figure 3.

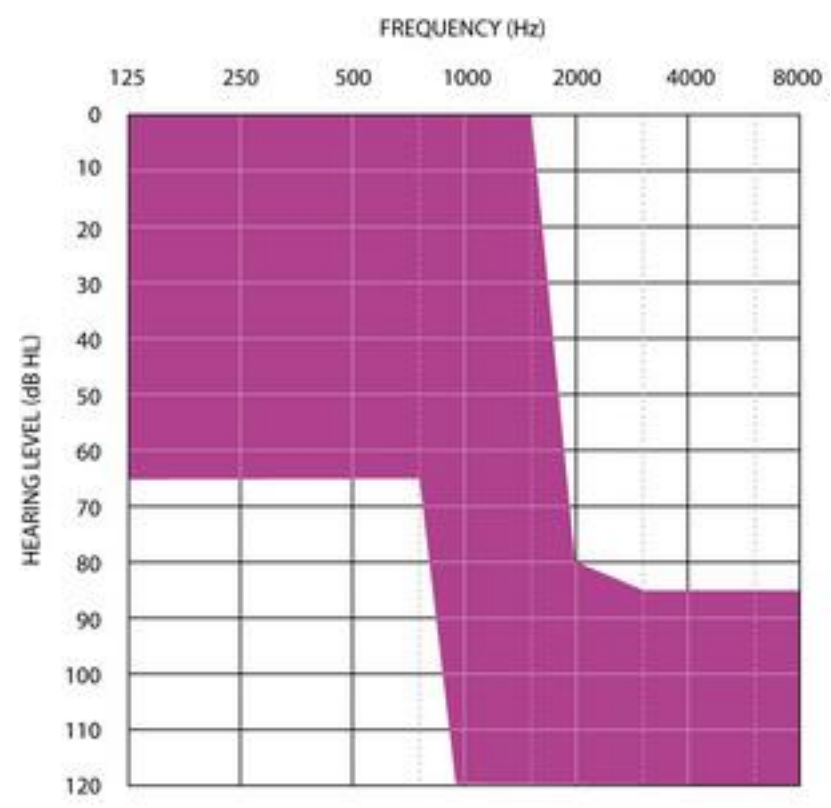

Figure 3: Candidacy criteria for Hybrid cochlear implantation

Figure used with permission of MED-EL (V. Lutz, personal communication, January 2014) 


\subsubsection{Typical hearing loss}

As previously mentioned, EAS candidates typically have a sloping severe to profound high frequency hearing loss with residual hearing in the low frequencies. The degree of hearing in the low frequencies can range from normal hearing to moderately severe hearing loss. Even though candidacy criteria do not exclude individuals with normal low frequency hearing, it is important to note that the majority of individuals who have been implanted with hybrid devices to date do have some degree of hearing loss in the low frequencies. Two of the three EAS patients in a study by Turner et al. (2004) had unaided thresholds of 60-65 dB HL for frequencies $500 \mathrm{~Hz}$ and below. Seven of the fourteen cases described in a study by Luetje, Thedinger, Buckler, Dawson, and Lisbona (2007) presented with a pre-operative pure-tone average $>60 \mathrm{~dB}$ HL between $250 \mathrm{~Hz}-1000 \mathrm{~Hz}$. Inclusion criteria for a clinical trial of the Iowa/Nucleus Hybrid implant included hearing loss of $>80 \mathrm{~dB}$ for frequencies above 2000 $\mathrm{Hz}$ and hearing thresholds up to $60 \mathrm{~dB}$ HL in the low frequencies (Turner et al., 2008). Turner et al. (2008) also indicated a mild but non-significant relationship between residual hearing thresholds and speech recognition threshold (SRT) as well as suggesting that the advantage of residual low frequency hearing appears to be preserved unless low frequency hearing loss approaches profound severity. Another important indication for EAS candidacy is low monosyllabic speech perception scores in the best aided condition, indicating insufficient benefit from acoustic amplification alone (von Ilberg et al., 2011).

The typical hearing thresholds of EAS candidates can be visualized in Figure 4 which shows the preoperative hearing thresholds of patients who participated in a clinical trial of a hybrid cochlear implant (Kiefer et al., 2005) 


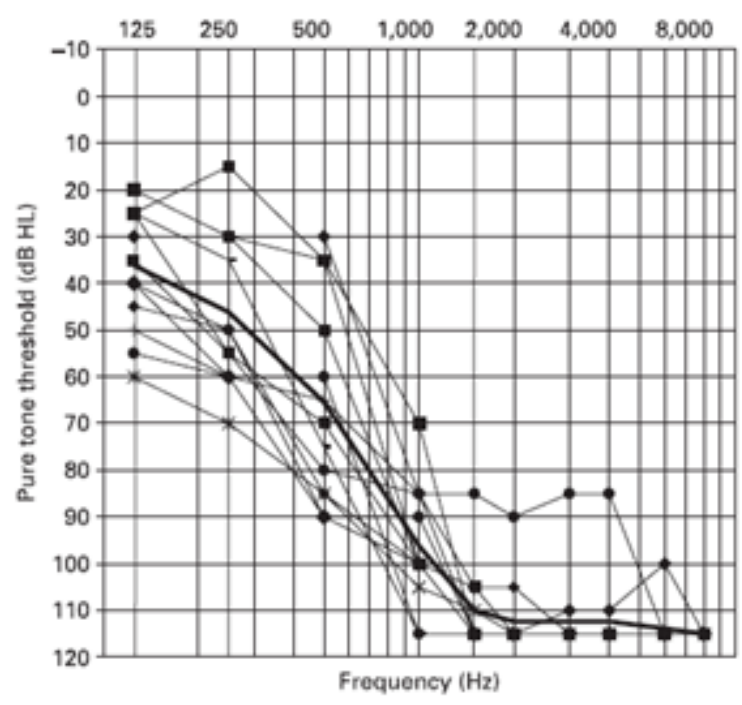

Figure 4: Pre-operative hearing thresholds of EAS candidates

As adapted from Kiefer et al. (2005), p137. Copyright (C 2005 Karger Publishers, Basel, Switzerland.

Based on the typical degree and configuration of hearing loss in EAS recipients, we can conclude that they are likely to suffer from outer hair cell loss/damage in the low frequencies. This accounts for the reduced hearing thresholds at these frequencies which could be mediated with a traditional hearing aid. The other consequence of outer hair cell loss/damage is reduced frequency selectivity which is likely to manifest as speech perception difficulties (Turner, 2006). In the high frequency range, however, the degree of hearing loss indicates likely inner hair cell loss/damage. This means acoustic amplification is unlikely to be beneficial in the high frequencies and supports the strategy of bypassing the hair cells and stimulating the cochlear nerve directly. 


\section{Benefits of combined EAS: A review of the literature}

In this chapter we outline the benefits reported for combined electric-acoustic hearing from the literature. Studies in which actual EAS recipients were recruited as well as simulation studies will be summarized for a number of outcome measures.

\subsection{Outcomes for Speech in quiet}

\subsubsection{In studies with actual EAS recipients}

Gstoettner et al. (2009) found speech recognition scores for sentences in quiet of $30 \%$ for a hearing aid alone condition, $75 \%$ for a cochlear implant condition, and $79 \%$ for an EAS condition. These results show a large improvement with cochlear implant listening over listening with a hearing aid alone, but very little additional benefit from combining the two.

Similar results were found by Kiefer et al. (2005) who reported improvements for sentences in quiet from $32 \%$ with hearing aids alone, to $78 \%$ with cochlear implants alone, to $86 \%$ with EAS. Scores were lower for a monosyllabic word recognition task due to differences in target stimuli such as reduced context. However, a similar pattern of results was evident with scores of $7 \%$ before implantation with hearing aids alone, which increased to $56 \%$ with the cochlear implant alone, and to $62 \%$ with EAS.

Helbig, Baumann, Helbig, von Malsen-Waldkirch, and Gstoettner (2008) reported no significant difference for speech in quiet for participants who switched over to a hybrid cochlear implant from a standard cochlear implant. They believed this was due to ceiling effects with participants attaining a $96 \%$ score with the cochlear implant only, compared to a 97\% score with the new bimodal device. A considerable limitation of this study was that in order to switch from a cochlear implant only device to a bimodal DUET device with both cochlear implant and hearing aid components, all participants had to have residual hearing in 
the low frequencies. Participants would therefore have received both electric and acoustic stimulation even prior to the switch-over, even though they might not have received additional amplification in the low frequencies.

The pattern of results from several clinical trials showed a similar finding. Recipients consistently seem to achieve much higher speech recognition in quiet with cochlear implants as compared to with hearing aids alone. Combined EAS often added to this benefit, but not always significantly so. This finding is interesting as it suggests the additional benefit obtained from combining electric and acoustic hearing may be negligible for speech in quiet. Furthermore, these results seem to allude that even if residual hearing preservation was not achieved following cochlear implant surgery, individuals who meet the current EAS inclusion criteria are still likely to achieve improved outcomes for speech in quiet with a cochlear implant as compared to their pre-implant performance using acoustic amplification.

\subsubsection{In simulation studies}

EAS listening can be simulated by combining vocoded high frequency sound with unprocessed low frequency sound. Seldran et al. (2010) measured syllable identification in disyllabic words presented in quiet to 24 normal hearing listeners while adjusting the following parameters as part of their EAS simulation: (1) mode of stimulation, (2) cut-off frequency between vocoded sound and low-pass filtered acoustic sound, and (3) number of channels of vocoder. They found that speech recognition in quiet was superior for the EAS condition compared to recognition in either acoustic or electric alone conditions. They also reported that 4 channels were sufficient for good syllable identification and that recognition scores improved with increasing cut-off frequency between electric and acoustic hearing. This latter finding seems to imply that the higher the frequency at which a hybrid CI recipient is still able to utilize acoustic hearing, the better their speech recognition may be. 
Arehart, Souza, Muralimanohar, and Miller (2011) investigated the effects of age on listeners' ability to utilize fundamental frequency differences in order to obtain benefit from simulated EAS listening. One group of younger and one group of older normal hearing listeners participated in tasks of multiplicity, in which they had to say whether a stimulus was comprised of 1 or 2 sounds, and double-vowel identification, in which they had to identify competing vowels. Arehart et al. (2011) found that fundamental frequency differences facilitated performance for both the multiplicity and the double-vowel identification tasks in the EAS condition. They also found that there was large inter-subject variability between both older and younger listeners in their ability to use fundamental frequency differences to integrate and benefit from combined EAS hearing. They commented that this alludes to the role of higher level processing in an individual's ability to benefit from combined EAS.

\subsection{Outcomes for speech in noise}

Although some benefit has been reported for speech in quiet, this is much less than the benefit that has been reported for speech in noise with EAS. It is believed the spectral resolution achieved with cochlear implants is sufficient for good speech perception in quiet (Friesen et al., 2001). However, when background noise is present the additional fine structure spectral and temporal cues offered by the acoustic hearing in the low frequencies will likely have a significant impact on speech recognition.

\subsubsection{In studies with actual EAS recipients}

In a group of subjects participating in the FDA clinical trial of the Iowa/Nucleus hybrid cochlear implant, Gantz et al. (2005) found a $9 \mathrm{~dB}$ improvement in speech recognition in noise with combined electric and acoustic hearing as compared with standard cochlear implant recipients matched for speech recognition in quiet. Ongoing results of the Iowa/Nucleus clinical trial indicated that nearly all subjects had improved word 
understanding compared to pre-operative hearing, with word understanding continuing to improve more than 24 months after implantation (Gantz et al., 2006). Furthermore, when matched to long-electrode implant users for word understanding in quiet, the Hybrid group showed a mean advantage of $6.1 \mathrm{~dB}$ in multi-talker babble and $7.5 \mathrm{~dB}$ in steady state noise. Turner et al. (2008) found a $9 \mathrm{~dB}$ advantage for speech in noise performance when Hybrid implant users were compared to a large group of traditional long-electrode users. When this group of Hybrid implant users were compared to a smaller group of long-electrode users matched by their speech recognition in quiet, a $4.2 \mathrm{~dB}$ advantage was observed. For individual patients in a prospective study by Kiefer et al. (2005) benefits of more than $70 \%$ for EAS stimulation as compared to cochlear implant alone was found, indicating a strong synergistic effect of EAS for some patients. Turner, Gantz, Karsten, Fowler, and Reiss (2010) conducted a comparison study between a group of hybrid cochlear implant users and traditional long-electrode cochlear implant users. They investigated speech understanding in a background of other talkers between the two groups, as well as the patient factors which seemed to predict the probability of success with EAS. They found that the Hybrid cochlear implant group outperformed the traditional cochlear implant group by $5 \mathrm{~dB}$ on average for speech recognition thresholds for spondee words in a background of other talkers. Furthermore, they found age at implantation and duration of hearing loss to be predictive factors for success with EAS stimulation. In their study, increasing age resulted in reduced performance, particularly in regards to electrical stimulation, which they hypothesised might be due to more difficulty with cortical adaptation in elderly patients. They also found that the longer the duration of hearing loss, the poorer the performance, which is most likely due to auditory deprivation and degeneration of auditory nerves.

Similar findings for speech in background noise with combined EAS have been reported by several other authors (Dorman, Gifford, Spahr, \& McKarns, 2008; Helbig et al. 
2008; Kong, Stickney, \& Zeng, 2005). These results consistently seem to indicate that by combining acoustic and electric stimulation, individuals are able to achieve better speech recognition in noise than they can with either of the stimulation modes in isolation.

\subsubsection{In simulation studies}

In addition to studies with actual EAS recipients, the potential benefit of EAS has also been extensively examined in a series of simulation studies, in which vocoded high frequency information is combined with unprocessed low frequency information and presented to normal hearing listeners (Arehart et al., 2011; Seldran et al., 2010). The results of these studies have been largely consistent with those observed in hybrid cochlear implant recipients, in that a significant benefit of combined electric and acoustic hearing is evident, particularly for speech recognition in a background of competing speech (Brown \& Bacon, 2009; Chang, Bai, \& Zeng, 2006; Dorman, Spahr, Loizou, Dana, \& Schmidt, 2005; Li \& Loizou, 2008; Qin \& Oxenham, 2006; Turner et al., 2004).

In a simulation study by Turner et al. (2004), a significant advantage of simulated combined EAS listening over a 16-channel full-electrode simulation was found for speech recognition in a background of other talkers, but not in steady-state noise. A similar finding was reported by Li and Loizou (2008) who also observed a significant advantage when combining low-pass filtered speech with vocoded speech to simulate EAS hearing for a background of competing talkers, but not for steady state noise.

This is somewhat in contrast to findings from Qin and Oxenham (2006), and Brown and Bacon (2009) who observed this advantage of simulated EAS over simulated longelectrode implantation in both competing-talker and steady-state noise background. However, it should be noted that Brown and Bacon (2009) chose not to include the background in the low frequency region which might account for this discrepancy. In their study, Brown and 
Bacon (2009) examined the effect of replacing the target speech in the low-frequency region with a tone modulated in either frequency, amplitude or both to match dynamic changes in the target talker's fundamental frequency and envelope of the low-pass speech. This was done in order to investigate the contributions of each of these cues in the acoustic region to combined EAS benefit. They found a significant benefit over a vocoder alone condition with the addition of a tone carrying either fundamental frequency or amplitude envelope cues, and the greatest benefit when these were combined. The magnitude of combined EAS benefit when both fundamental frequency and amplitude envelope cues were provided was as much as 57 percentage points over the vocoder alone condition.

Qin and Oxenham (2006) conducted an EAS simulation study in which they measured speech perception for sentences with a background of either steady-state speech shaped noise or a single background talker. The added low frequency information was low-passed at either $300 \mathrm{~Hz}$ or $600 \mathrm{~Hz}$. They found that although steady-state speech shaped noise was a more effective masker for the condition in which target speech was unprocessed, this effect of background masker was not present for the vocoder alone or EAS conditions. Scores were not significantly different between the maskers for these conditions, indicating that the benefit when adding low-pass speech to vocoded high frequencies was present for both steady-state speech shaped noise and a single background talker. Furthermore, they found that significantly improved speech recognition scores were obtained for conditions where low-pass speech was added to vocoded speech to simulate EAS as compared to vocoded speech alone. This improvement was largest for the higher cut-off frequency of $600 \mathrm{~Hz}$, but a $2.5 \mathrm{~dB}$ improvement in speech recognition threshold was observed even when low-pass speech below $300 \mathrm{~Hz}$ was added to the vocoded high frequency region. This left the authors “cautiously optimistic" (Qin \& Oxenham, 2006, p. 2424) about the possibilities of EAS even for individuals with very limited low frequency residual hearing. 
Dorman et al. (2005) investigated the effect of insertion depth by leaving varying sized 'gaps' in frequency between low frequency acoustic hearing and simulated high frequency electric hearing in normal hearing subjects. Not surprisingly they found that as the gap size decreased, intelligibility increased. They also wanted to determine if their EAS simulation captured what they refer to as the 'EAS signature', whereby speech in noise scores for combined EAS are greater than the sum of the scores in each stimulation mode alone. This was certainly the case in their study. The EAS condition score (90\%) was not significantly higher than the sum of the acoustic-only (17\%) and electric-only (71\%) conditions when there was a gap of $0.5 \mathrm{kHz}$ between the vocoded high frequency sound and the low-pass sound. For larger gaps $(1 \mathrm{kHz}, 1.5 \mathrm{kHz}, 2.2 \mathrm{kHz})$ this synergistic effect in which scores for combined EAS is significantly higher than the sum of each stimulation mode alone was present.

It is interesting to note that as observed in both studies with actual EAS recipients and simulation studies, the EAS benefit for speech in noise can be present even when acoustic stimulation alone provides no speech intelligibility. This was demonstrated by Chang et al. (2006) who found a functional improvement in SNR to be present even when low frequency sound below $250 \mathrm{~Hz}$ was combined with a cochlear implant simulation. Qin and Oxenham (2006) reported a similar finding.

\subsection{Outcomes for Melody Recognition and Subjective sound quality}

Although seldom included as an outcome measure in EAS clinical trials, self-reported anecdotal evidence seem to indicate that recipients of combined EAS experience improved melody recognition and subjective sound quality (Gantz et al., 2006). Improved voice recognition has also been mentioned as a likely benefit of this type of stimulation. It is believed this is due to the preservation of fine spectral information in the low frequencies 
necessary for pitch discrimination and music appreciation. Melodies are made up of a series of pitch patterns, therefore, the relatively poor performance on pitch perception tasks reported for cochlear implant recipients (Gfeller et al., 2002) is likely to have an impact on their ability to both recognize and appreciate melodies and music. Furthermore, the qualities of sound produced by cochlear implants have been described as 'raspy' and 'mechanical'. The quality of sound that can be achieved when acoustic hearing is present is much more natural (Turner et al., 2010). Subjectively patients have reported perceiving electric-acoustic stimulation as a single united modality of hearing, rather than being able to hear two differing sound modalities, hinting towards cortical integration of the two modes of stimulation leading to a single perceptual experience (Kiefer et al., 2005).

Interestingly a study by Kong et al. (2005) in which 5 cochlear implant recipients with residual hearing in the non-implanted ear participated in a melody recognition experiment revealed no significant difference in ability to recognize familiar melodies between the hearing aid alone, cochlear implant alone, and EAS conditions. This was in contrast to a finding by Gantz et al. (2005) in which five short-electrode recipients achieved a score of $80 \%$ for familiar melody recognition compared to a score of only $31 \%$ achieved by traditional long-electrode cochlear implant recipients.

Helbig et al. (2008) found no significant difference in scores for the Abbreviated Profile of Hearing Aid Benefit (APHAB) before and after participants were switched over from a cochlear implant only to a bimodal hybrid cochlear implant device. The APHAB is commonly used as an assessment of subjective benefit achieved with amplification. A nonsignificant result implies that the participants in this study did not experience reduced difficulty with their new hybrid cochlear implant device compared to their traditional cochlear implant. As previously pointed out it should be noted that all participants in this study had residual hearing in the low frequencies prior to switch-over. Thus they would have 
received both electric and acoustic stimulation prior to switch-over, even though they might not have received additional amplification in the low frequencies. This limitation might account for the non-significant results in regards to the APHAB.

\subsection{Underlying mechanisms of EAS benefit in noise}

We know that being able to combine both electric and acoustic hearing results in better speech in noise recognition than either modality in isolation. The precise mechanism of this phenomenon, however, is not clear. There are several differences between acoustic and electric hearing that might account for the release from masking when acoustic stimulation is combined with electric stimulation. One of these is improved spectral resolution. Spectral resolution is the ability to resolve frequency components within a signal and relates to the accuracy of pitch perception. Pitch perception in cochlear implants is limited in part by the

number of distinct 'place-frequency' bands they have available to them. This limitation is not only due to the number of electrodes but also due to the current spread of those electrodes within the usually finely tuned tonotopic cochlea. Current spread is the phenomenon whereby a large region of nerve fibres are stimulated once current is discharged from the electrode of the cochlear implant (Brown \& Bacon, 2010). This means that electrodes in very close proximity to one another would likely stimulate the same nerve fibres and therefore be indistinguishable from one another. In other words, even if manufacturers were to place increasing numbers of electrodes on current cochlear implant electrode arrays, recipients are unlikely to experience improved spectral resolution (Friesen et al., 2001). Not only is spectral resolution limited with cochlear implants, but encoding of spectral information might also be inaccurate and imprecise due to the potential place-frequency mismatch between the electrodes and the tonotopically arranged cochlea. This reduced spectral resolution doesn't seem to be a large limitation for speech in quiet, but has a big effect on speech in noise and unfortunate effects on the aesthetic quality of music (Gantz et al., 2005) Another difference 
between acoustic and electric hearing is improved temporal resolution. Temporal resolution relates to the ability to identify changes in the signal in the time domain. The electrical pulses delivered by electrodes to the auditory nerve in cochlear implant processing are modulated by the temporal amplitude envelope. This means that temporal fine structure is not transmitted (Stickney, Assmann, Chang, \& Zeng, 2007). The temporal envelope information delivered via cochlear implants appears to be sufficient for speech in quiet, but the fine structure cues available with acoustic hearing may improve temporal pitch information required for speech in noise. This improved temporal processing ability for acoustic hearing compared to electric hearing has been identified as a potential explanation for the EAS benefit in noise (Kong et al., 2005).

An investigation by Golub, Won, Drennan, Worman, and Rubinstein (2012) shed some light on the underlying mechanisms that might be responsible for the EAS benefit in noise. They compared spectral-ripple discrimination, Schroeder-phase discrimination, and temporal modulation detection between a group of hybrid cochlear implant participants and results previously reported for traditional long-electrode cochlear implant listeners. These psychoacoustic tests were chosen as they assess some of the specific perceptual differences between electric and acoustic hearing, as well as being well correlated with the clinical performance of cochlear implant listeners. A spectral ripple stimulus is one in which amplitude rises and falls systematically as a function of frequency. Spectral-ripple discrimination assesses an individual's ability to distinguish a stimulus with an inverted ripple from a standard ripple. A Schroeder-phase stimulus is a harmonic tone in which lead or lag accumulates systematic as a function of harmonic number. Schroeder-phase discrimination assesses an individual's sensitivity to rapidly changing temporal cues by investigating their ability to discriminate one phase sequence from the other. Temporal envelope sensitivity was assessed using a temporal modulation detection task during which 
the subjects had to identify the interval containing sinusoidally amplitude modulated noise. Participants also completed the University of Washington Clinical Assessment of Music Perception Test (UW-CAMP) and a speech reception in steady state noise test. Results showed that hybrid cochlear implant users performed significantly better on the spectralripple discrimination test and the pitch-direction discrimination portion of the UW-CAMP. There was no significant difference between the groups for the Schroeder-phase discrimination test and the temporal modulation detection test. These results led the authors to conclude that improved spectral information, rather than temporal information, is the likely candidate for improved speech recognition in noise when combining electric and acoustic stimulation. Despite a small sample size $(n=5)$ this study provides valuable insight into the speech in noise benefit obtained with combined EAS.

In addition to not being certain which perceptual difference accounts for the EAS benefit in noise, the method of integration of the two modes of stimulation or the way in which the target signal is separated from the background noise is also not understood. For a number of years it was assumed that the improved fine pitch information achieved with the low-frequency acoustic stimulation can be combined with the relatively weak pitch information from the electric stimulation to account for the EAS benefit in noise. Of particular interest has been the role of the fundamental frequency, which is poorly conveyed by cochlear implants but accurately represented by the fine spectral and temporal acoustic cues available with residual low frequency hearing (Brown \& Bacon, 2010; Sheffield \& Zeng, 2012). Qin and Oxenham (2005) demonstrated the reduced ability to use fundamental frequency to segregate competing signals in a simulation of cochlear implant listening. Several authors have hypothesised that the improved perception of fundamental frequency of the target speaker could be used to segregate the target speech from the interfering background noise in electric-acoustic hearing (Chang et al., 2006; Kong et al., 2005; Qin \& 
Oxenham, 2006; Turner et al. 2004). This seems a logical assumption since the role of fundamental frequency and pitch cues for auditory stream segregation has been clearly demonstrated in normal hearing listeners (Assmann \& Summerfield, 1990). Further evidence was provided by Kong et al. (2005) who demonstrated significantly better speech recognition performance for 4 cochlear implant recipients with residual acoustic hearing in the nonimplanted ear when the speech masker was a voice of a different gender to the target speaker. This indicated that as fundamental frequency differences increased so did the ability to segregate the two competing stimuli. Further evidence in support of this hypothesis was provided by Chang et al. (2006) who demonstrated the EAS benefit when low-pass speech (below $300 \mathrm{~Hz}$ ) contained the fundamental frequency only.

Kong and Carlyon (2007) were the first to dispute auditory stream segregation as the underlying mechanism of EAS benefit. In a simulation study which involved normal hearing listeners listening to vocoded speech in one ear and low-pass filtered speech in the other, they found a combined EAS advantage was maintained at $5 \mathrm{~dB}$ SNR even when fundamental frequency contour cues were removed. This was done by replacing the fundamental frequency contour cues of the target speech with a harmonic complex with a fixed fundamental frequency of $150 \mathrm{~Hz}$, and then modulating this fixed harmonic complex with the amplitude envelope of the voiced portions of the target speech. They determined that voicing and amplitude envelope cues accounted for this EAS benefit, not fundamental frequency cues. They argued that this suggests a glimpsing mechanism may in fact account for the EAS benefit in noise. Glimpsing refers to the phenomenon by which listeners take advantage of momentarily improved signal to noise ratios occurring within the 'dips' of a fluctuating masker such as speech. This might occur in times when the level of the target is relatively high or when the level of the masker is relatively low. The idea is that the additional acoustic information such as voicing in the low frequencies provides a cue for the listener of when to 
'glimpse' the target. This is thought to allow listeners to extract relevant information regarding the target signal at fluctuating intervals thereby improving speech perception. Brown and Bacon (2009) also supported glimpsing as the underlying mechanism for EAS benefit in noise. However, in contrast to Kong and Carlyon (2007) they found both fundamental frequency and amplitude envelope to be useful cues, with each cue proving more important than the voicing cue for EAS benefit. They believed their results argued against the use of fundamental frequency for source segregation as benefit was independent of the magnitude of fundamental frequency differences between the target and the background speakers, and the addition of background speaker cues such as amplitude envelope did not increase the observed advantage. A similar finding was reported by Stickney et al. (2007) for both cochlear implant recipients and normal hearing listeners listening to a 24-channel vocoded cochlear implant simulation. They found no benefit for speech recognition in background noise with increasing fundamental frequency separation between target speech and background speech. These results are in contrast to those of Kong et al. (2005), and Cullington and Zeng (2008), who did find improved speech recognition with increasing voice pitch differences between the target and the background speakers. Other researchers have also supported the glimpsing account for EAS benefit in noise (Li \& Loizou, 2008).

Interestingly, although Turner et al. (2004) and Li and Loizou (2008) reported similar results, showing that the EAS benefit was present for speech in a background of other talkers, but not in steady-state noise, the authors attributed these results to different mechanisms. Turner et al. (2004) concluded that the observed benefit in a background of other talkers as compared to steady-state noise supports the role of fundamental frequency for auditory stream segregation as the differences between the fundamental frequencies of the speakers can be utilized to separate target speech from background speech. Li \& Loizou (2008) on the 
other hand drew the conclusion that these results demonstrate the important role of glimpsing, as glimpsing requires a fluctuating masker such as speech with temporal envelope 'dips' which is lacking in steady-state noise. Both these hypotheses seem reasonable and the results do not appear to provide clear evidence for one underlying mechanism over the other.

Another possibility proposed by Sheffield \& Zeng (2012) is that the low frequency acoustic signal provides information about the target speech itself which assists speech recognition. They believed this might occur instead of, or in addition to glimpsing and/or sound source segregation. They provided evidence for this hypothesis by showing that the addition of amplitude modulated fundamental frequency improved consonant perception for simulated cochlear implant speech in quiet and in steady-state noise. Since neither background involves fluctuating maskers they argued the observed EAS benefit cannot be exclusively due to glimpsing or segregation, but rather that a separate mechanism is responsible.

\subsection{Comparison of outcomes between simulation studies and studies with actual EAS recipients}

Despite the utility of simulation studies, particularly for a treatment that is controversial and invasive, the limitations of simulation in a normal hearing population should not be underestimated. These limitations are clearly evident when we consider the discrepancy in performance between individuals in an EAS simulation study and real EAS recipients.

A study by Turner et al. (2004) provides a good point of comparison as they had both simulated EAS listeners as well as real EAS recipients in their study which means the same methodology was applied to both participant groups. They tested spondee word identification in a competing talker background and found a speech recognition threshold of 
approximately -20 dB SNR when electric-acoustic stimulation was simulated in a group of normal hearing subjects. The same spondee word identification task was also completed by a group of 3 patients implanted with a short-electrode hybrid cochlear implant device as part of another experiment. For this group of participants the speech recognition threshold was approximately -8dB SNR. In other words, there was a speech recognition threshold difference of nearly $12 \mathrm{~dB}$ between normal hearing listeners in which electric-acoustic stimulation was simulated and individuals who had undergone hybrid cochlear implant surgery.

A recent study by Yang and Zeng (2013) in which they attempt to replicate a simulation study by Qin and Oxenham (2006) in actual EAS recipients delivered interesting and surprising results. They found that for both the acoustic alone condition and the electric alone condition the performance was similar between the actual EAS recipients and the simulation participants. Despite this, for the combined EAS condition, performance of actual EAS recipients was 21 percentage points below that of simulated EAS listeners, indicating a significant decrease in efficiency of integrating acoustic and electric information for actual EAS recipients. The authors concluded that these results indicated an overestimation of the synergetic integration of acoustic and electric stimulation.

One has to be cautious when drawing comparisons between simulation studies and studies with actual EAS recipients. This is because of the differences in outcome measures, test signals, procedures, conditions and overall methodology between various studies. Except for the study by Turner et al. (2004) in which the study contained both participant groups, and the study by Yang and Zeng (2013) that was carried out specifically as a point of comparison, it was not felt that further comparisons could accurately or fairly be drawn. Nonetheless, even these studies are enough to indicate a discrepancy in speech recognition outcomes between EAS simulations in normal hearing listeners and actual EAS recipients. It is our hope that by 
simulating low frequency hearing similar to that of real EAS recipients, we will be able to reduce at least some of the variation between these two methods of investigation.

There are several speculated reasons for the improved speech recognition scores obtained in EAS simulation studies compared to studies in which actual EAS recipients were recruited. One example is auditory deprivation. Due to reduced auditory input over time it is possible that cochlear implant recipients do not have the full population of surviving auditory neurones that normal hearing listeners are expected to have. Yang and Zeng (2013) found a correlation between EAS integration efficiency and duration of deafness in the implanted ear, supporting the importance of early implantation and the potential risk of auditory deprivation. The reduced performance for real EAS recipients may also be related to the place-frequency mismatch that might occur due to electrode insertion. Yang and Zeng (2013) identified some further important caveats of simulation studies. They pointed out that vocoded speech is thought to simulate the best cochlear implant performance, not average performance, meaning vocoded simulation studies are likely to be representative of only the best possible outcomes with this type of processing. They also commented on the role of an individual's hearing experience. Previous listening experiences are likely to have an impact on how the central auditory system processes sound. Last but not least they argued that real EAS users are likely to have some hearing loss in the low frequencies whereas simulation studies often leave low frequency information unprocessed, thus simulating ideal hearing in the low frequency region. This caveat is of particular relevance to the current study as low frequency hearing in this study will be processed to simulate varying degrees of hearing loss, thus addressing this limitation. 


\section{Overview of auditory filters}

\subsection{The role of auditory filters in normal hearing}

The auditory filter is a psychophysical construct that underlies frequency analysis of sounds. Frequency analysis is perhaps one of the most important abilities of the human auditory system as it allows listeners to discriminate between different sounds based on their frequency spectra. In other words, being able to tell the difference between a tone at $300 \mathrm{~Hz}$ and a tone at $400 \mathrm{~Hz}$ is the result of the frequency analysis/selectivity of our auditory system. Spectral components which fall within the same auditory filter are said to be unresolved. The ability to distinguish one frequency from another depends on the patterns of excitation in different auditory filters.

It is clear therefore, that auditory filter width is a crucial factor in the ability of the auditory system to analyse spectral information. In other words, as the auditory filter width increases, the frequency selectivity decreases. A number of psychophysical methods can be used to measure the auditory filter shape and width in humans. The most commonly used of these methods is the notched-noise method described by Patterson (1976). This method involves measuring the threshold of a tone centred at a fixed frequency while varying the bandwidth of a notched-noise, with the notch centred at the tone frequency. As seen in the top panel of Figure 5, the auditory filter typically assumes a roughly symmetrical shape with a rounded top and steep skirts. It is believed that a series of partially overlapping auditory filters of said shape is responsible for frequency selectivity in the peripheral auditory system (Moore, 2013). The tuning and frequency to place map of the basilar membrane is the first stage of processing that determines the auditory filter, but central auditory processing is also involved. Patterson's method has extensively been used to measure the equivalent rectangular bandwidth (ERB) of auditory filters, which has typically shown ERBs between $11 \%$ and $17 \%$ 
of the centre frequency (Moore, 2013). The ERB as a function of centre frequency, $\mathrm{F}$ in $\mathrm{kHz}$, for normal hearing listeners can be described by the equation:

$\mathrm{ERBn}=24.7(4.37 \mathrm{~F}+1)($ Glasberg \& Moore, 1990)

Another important consideration for auditory filter width is the relative effect of masking. Masking occurs when the audibility of one sound is decreased by the presence of another sound. This consideration is important as the current experiment involves a signal in the background of other sounds. The current assumption is that when detecting a signal in the presence of background noise, noise falling within the auditory filter centred around the signal frequency has the ability to mask the signal. Noise which falls outside the bandwidth of the auditory filter containing the target signal is attenuated. We can deduce, therefore, that as the width of the auditory filter increases, so does the effect of the noise. A decreased signal to noise ratio within the auditory filter will decrease the individual's ability to detect the target signal. The width of the auditory filter is crucial not only for accurate frequency analysis, but also for reducing the effect of masking on a target signal.

\subsection{The effects of sensorineural hearing loss on auditory filters}

It is known that while inner hair cells are crucial for perception of sounds at different frequencies, it is the outer hair cells that 'fine tune' this frequency response. It is clear that the sharpness of this finely tuned frequency response decreases when there is damage to the outer hair cells, as occurs in sensorineural hearing loss. This cochlear damage typically manifests itself as auditory filters which are significantly broader, more asymmetrical, and more irregular than would be expected for normal hearing listeners. This was well demonstrated by Glasberg and Moore (1986) who measured the auditory filter shapes of 5 individuals with unilateral hearing loss due to cochlear damage. Figure 5 show the auditory filters at $1 \mathrm{kHz}$ for the normal ears as plotted in the upper panel and the auditory filters at $1 \mathrm{kHz}$ for the impaired 
ears as plotted in the lower panel. It is clearly illustrated in Figure 5 that impaired ears produced significantly broader auditory filters with more pronounced asymmetry.

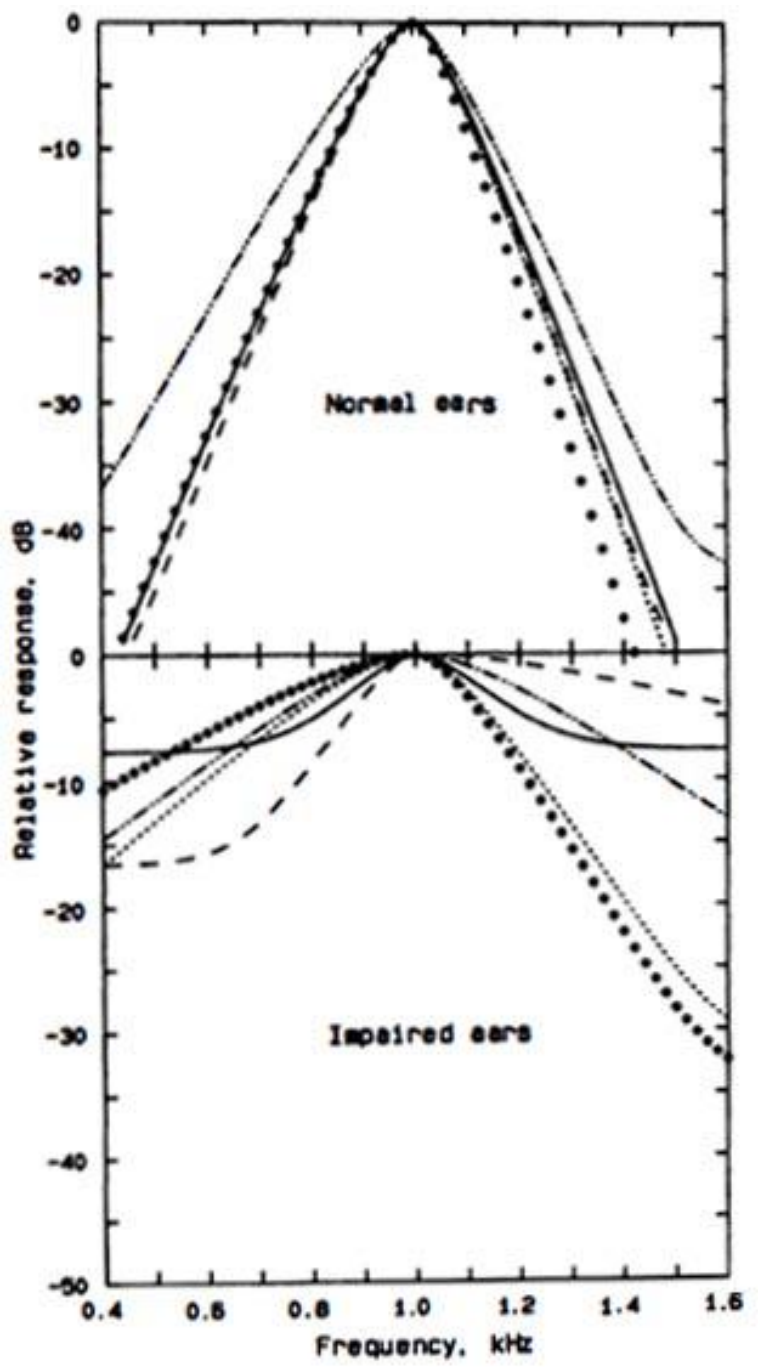

Figure 5: Derived auditory filter shapes at $1 \mathrm{kHz}$ in normal and impaired ears

As adapted from Glasberg and Moore (1986)

As mentioned previously this will not only have the effect of reducing the auditory system's frequency selectivity, but also to increase the effect of masking. Furthermore, reduced frequency selectivity is likely to impair an individual's perceptual analysis of more complex signals such as speech and music, which requires fine spectral analysis (Moore, 2013). 


\subsection{Overview of a method used to simulate broadened auditory filters}

It is possible to simulate broadened auditory filters in normal hearing listeners through a method known as spectral smearing, described by Moore, Glasberg, and Simpson (1992). The aim of spectral smearing is to evoke excitation patterns in a normal ear similar to that evoked in an impaired ear by 'smearing' the spectral information of a signal. In short, spectral smearing is done by first dividing the target signal into frames in the time domain and then using the short-term fast Fourier transform (FFT) to transform each frame into amplitude and spectral information. The signal can then be smeared by modifying the spectrum of the signal, after which the inverse FFT can be used to transform the modified spectra back into a speech signal. The signal is re-synthesized by adding the waveforms in each frame using the overlap-add method (Allen, 1977; Baer \& Moore, 1993). Typically, successive frames overlap each other by half the frame length. In our study each frame was $20 \mathrm{~ms}$ duration and each successive frame overlapped by $10 \mathrm{~ms}$, resulting in $50 \%$ overlap. The effect of spectral smearing on stimuli in the current study can be visualized in Figure 6 which shows the consequences of spectral smearing at a factor of $\mathrm{x} 3$ as applied to a single vowel isolated from a sentence in quiet. As illustrated in this figure, the smeared spectrum appears to have reduced spectral information and smoothing of spectral peaks at 1.3, 2.0, and $3.5 \mathrm{kHz}$ which appear less pronounced in the smeared spectrum compared to the original spectrum. 

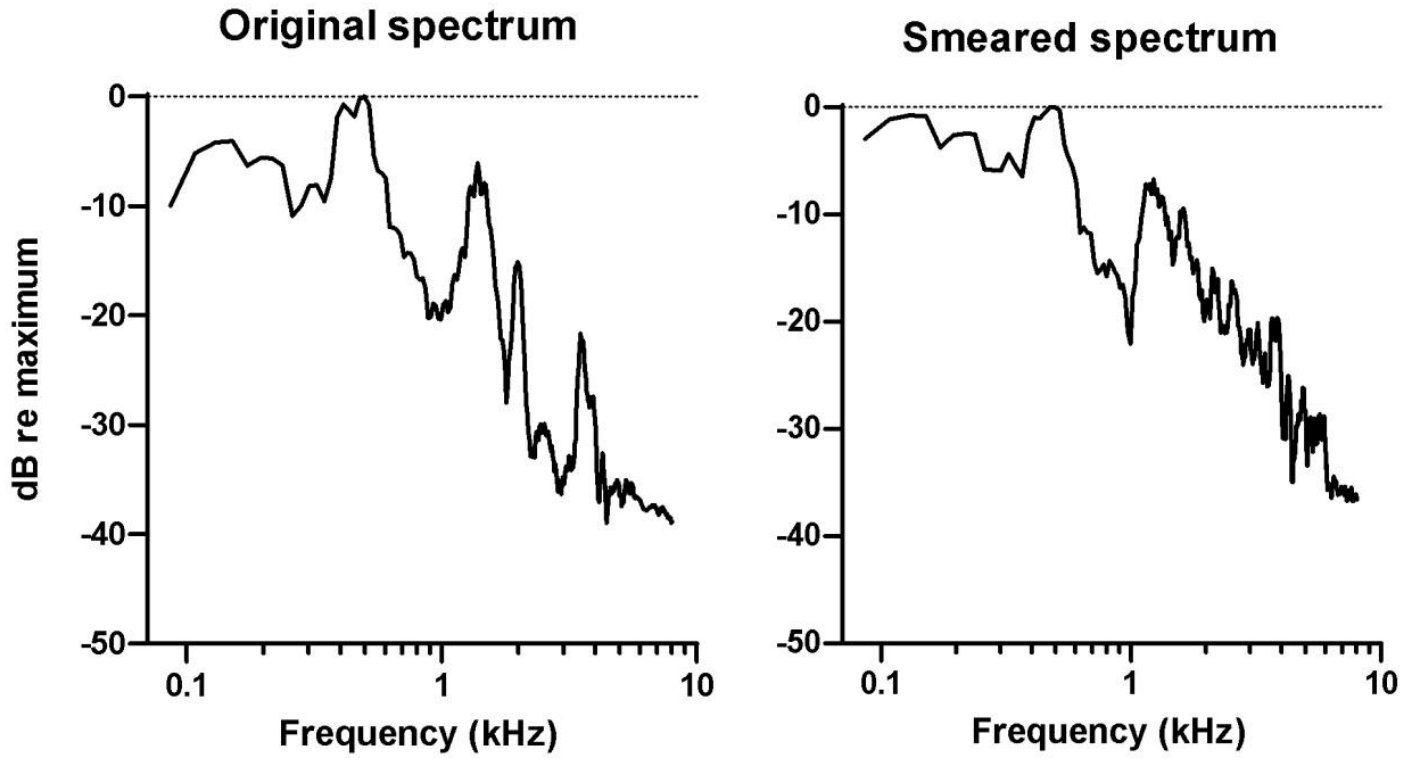

Figure 6: Spectral smearing applied to a single vowel isolated from a sentence in quiet, as applied in the current study

A study conducted by Baer and Moore (1993) in which spectral smearing was applied to sentences in quiet and in speech-shaped noise showed that spectral smearing had very little effect on speech intelligibility in quiet, even when auditory filters six times broader than normal were simulated. In a noise background, however, spectral smearing had a significant impact on speech intelligibility, with speech recognition scores decreasing as the auditory filter width increased. Speech recognition scores also decreased as the signal to noise ratio decreased. This is consistent with reported observations of individuals with cochlear hearing loss. Baer and Moore (1993) used spectral smearing factors of x3 and x6 as these degrees of broadening are thought to be representative of the auditory filter widths of individuals with mild-moderate and moderate-severe cochlear hearing loss respectively (Glasberg \& Moore, 1986). A follow-up study by Baer and Moore (1994) in which they investigated the effect of spectral smearing on speech intelligibility with a single talker background showed similar results. 


\section{Overview of current study}

\subsection{Rationale}

Although simulation studies are thought to be less representative of every-day functional performance, they are beneficial in that many of the complex extraneous variables often observed in studies with real cochlear implant listeners, such as duration of hearing loss and previous linguistic input, can be controlled for. This allows experimenters to alter different parameters and test them in isolation which would not be feasible in studies with actual cochlear implant participants. It can also be useful in overcoming the obstacle of having a limited target subject population available for recruitment, particularly in the early stages of investigating an invasive treatment such as EAS. Room for improvement of current EAS simulation studies has been identified by Qin and Oxenham (2006). They state that: "However, it is important to note that our simulations involve 'ideal' residual hearing, with no hearing loss and accompanying effects, such as broadened auditory filters. These conditions are unlikely to hold in real EAS users." (Qin \& Oxenham, 2006, p. 2424). Li and Loizou (2008), and Yang and Zeng (2013) also identified this assumption of 'ideal' low frequency hearing as a limitation in EAS simulation studies. This provided justification for the current study as we aimed to simulate residual hearing more similar to that of real EAS users.

To date, low frequency sound has remained largely unprocessed in simulation studies. In reality, however, Hybrid cochlear implant recipients are likely to have not only elevated hearing thresholds as measured by conventional audiometry, but also reduced frequency selectivity, both of which are likely to manifest as difficulty with speech perception.

By applying spectral smearing and attenuation to the low frequency region, the current study simulated various degrees of impaired residual low frequency hearing in normal hearing listeners. Listening as though via electric stimulation will be simulated using 
vocoding of the speech spectrum, as used by Shannon et al. (1995) to simulate cochlear implant listening.

\subsection{Aim of this study}

The aim of this study was to examine the effects of spectral smearing and attenuation of low frequency information on the identification of vocoded speech in noise. This was done in order to investigate the benefit for speech recognition in background noise that is commonly reported for combined EAS hearing. This will be achieved by applying vocoding to the high frequency range and various degrees of spectral smearing and attenuation of the low frequency range in normal hearing listeners, in order to simulate EAS hearing.

\subsection{Hypotheses}

Hypothesis 1: It is hypothesized that spectral smearing and attenuation will have a detrimental effect on the recognition of vocoded speech in noise, with signals with a larger degree of spectral smearing and attenuation requiring a higher signal-to-noise ratio (SNR) to maintain intelligibility.

Hypothesis 2: (a) It is also hypothesized that there will be a significant benefit for speech recognition in noise when combining simulated acoustic and electric hearing, compared to simulated electric hearing alone. (b) Furthermore, it is anticipated that this benefit will be evident despite spectral smearing and attenuation.

\subsection{Significance}

It is believed that the current research will add to understanding of a clinicallyimportant problem: The degree of benefit Hybrid CI recipients might expect based on their residual hearing status. Although this question has been addressed by studies in which actual EAS listeners were recruited, caution has to be taken when generalizing results as a large 
number of extraneous variables are likely to affect an individual's performance outcomes following Hybrid CI surgery. Furthermore, this question has not been addressed by simulation studies to date, as low frequency information has remained largely unprocessed in these studies.

Hybrid cochlear implantation is still a relatively new treatment for severe-profound sloping high frequency hearing loss. Use of a simulation method that more accurately resembles the performance of 'real' Hybrid CI users is likely to increase the validity of the current study, as well as open the door for further research in this field. 


\section{Methods}

A psychophysical experiment intended to evaluate the effect of reduced frequency selectivity and increased audiometric thresholds on the benefit of residual low frequency hearing for recognition of speech in noise in simulated EAS hearing was conducted. Procedures involving human subjects were reviewed and approved by the University of Canterbury Human Ethics Committee.

\subsection{Participants}

Twenty adults with normal hearing (thresholds $<20 \mathrm{~dB}$ HL across $250-4000 \mathrm{~Hz}$ ) were recruited into this study. Pure Tone Audiometry was performed using a GSI 61 Clinical Audiometer, which was calibrated within the last two years, in order to assess hearing prior to testing. Subjects were recruited primarily from the University of Canterbury via informal emails, personal acquaintances and word of mouth. Participants were compensated for their time with a $\$ 30$ grocery store voucher. Participants ranged from 22 to 49 years in age, with a mean age of 26 years. There was a gender distribution of 7 males and 13 females.

\subsection{Stimuli}

The speech stimuli used in this study were obtained from the coordinate response measure (CRM) corpus (Bolia, Nelson, Ericson, \& Simpson, 2000). Each sentence was presented in the form: "Ready [call sign] go to [colour] [number] now." The full CRM corpus contains eight call signs, four colours and eight numbers, all spoken by eight different talkers of American English (four male and four female talkers). Due to its large set size, low linguistic variability, and low contextual cues; the CRM is considered an appropriate research tool for the investigation of speech recognition in background noise, particularly competing speech noise (Eddins \& Liu, 2012). 
The target sentences were spoken by a female talker, using the call sign 'Baron'. Sentences were presented with a background of 3 competing talkers. Competing sentences were also obtained from the CRM corpus. Competing talkers were male and produced a different 'call sign' to that of the female target talker. The onsets of competing sentences were staggered, rather than synchronised to the target sentence, in order to better simulate 'real-life' listening with a background of competing talkers.

\subsection{Signal Processing}

To simulate EAS hearing and thus hybrid cochlear implant listening the target signal was processed in a variety of ways. A method of speech processing similar to that of electric stimulation was simulated using methods for noise vocoding extensively used and described throughout the literature (Arehart et al., 2011; Dorman et al., 2005; Shannon et al., 1995). Electric hearing through a fully inserted cochlear implant was simulated using an eightchannel noise-excited vocoder. Using eighth-order Butterworth filters, speech stimuli were band-pass filtered into eight channels between $80 \mathrm{~Hz}$ and $6000 \mathrm{~Hz}$. The lower and upper cutoff frequencies for each channel were taken from Arehart et al. (2011) as shown in Table 1 below.

Table 1: Cut-off frequencies for cochlear implant simulation (Arehart et al., 2011)

\begin{tabular}{|l|l|l|}
\hline Channel & $\begin{array}{l}\text { Lower cutoff } \\
(\mathrm{Hz})\end{array}$ & $\begin{array}{l}\text { Upper cutoff } \\
(\mathrm{Hz})\end{array}$ \\
\hline 1 & 80 & 202 \\
\hline 2 & 202 & 384 \\
\hline 3 & 384 & 657 \\
\hline 4 & 657 & 1065 \\
\hline 5 & 1065 & 1675 \\
\hline 6 & 1675 & 2589 \\
\hline 7 & 2589 & 3955 \\
\hline 8 & 3955 & 6000 \\
\hline
\end{tabular}


Half-wave rectification followed by low-pass filtering at $300 \mathrm{~Hz}$ was used to extract amplitude envelopes in each channel. Each envelope was then used to modulate an independent noise carrier. After modulation, the noise was band-pass filtered a second time, and the RMS amplitude within each channel was adjusted to match its original level.

To simulate the EAS conditions, the speech stimuli were low-pass filtered at $661 \mathrm{~Hz}$. Spectral smearing and/or attenuation were applied to this band, as described below. After processing, this output was combined with channels four to eight of the original vocoder simulation to generate the sentences that were presented to listeners.

Broadened auditory filters were simulated using a spectral smearing algorithm similar to that described by Baer \& Moore (1993). Each target sentence was divided into 20-msec frames. A Hanning window was applied to the signal in each frame. The fast Fourier Transform (FFT) was calculated, and the short-term spectrum was separated into amplitude and phase components. The amplitude spectrum was smeared by weighting each component by the value of the auditory filter (Glasberg \& Moore, 1990). The weights for the roex auditory filter were determined by the equation:

$$
W(g)=(1+p g) \exp (-p g)
$$

where $g$ is the deviation from centre frequency $(f c)$ of the filter and $p$ is a parameter determining the sharpness of the filter (Patterson, Nimmo-Smith, Weber, \& Milroy, 1982). The value of the sharpness parameter $p$ can be calculated from equations 2 (Baer and Moore, 1993) and 3 (Glasberg \& Moore, 1990)

$$
\begin{aligned}
& p=4 f c / E R B \\
& E R B=24.7(0.00437 f c+1)
\end{aligned}
$$


With these equations, values of $p$ can be calculated for normal ERBs, or for ERBs with abnormal widths, as occur in sensorineural hearing loss. The original spectral amplitudes were replaced with values weighted by $W(g)$. A new waveform for that frame was synthesized using these amplitudes and the original phases. The frame was advanced by $10 \mathrm{msec}$, and the entire process was repeated. The new waveforms for each frame was summed using the overlap and add method (Allen, 1977).

To simulate various degrees of hearing loss, the low frequency band was also attenuated in Conditions 5 and 6. After spectral smearing and attenuation (if any), the processed low-frequency band was added to the vocoded upper band.

\subsection{Experimental Conditions}

The following signal processing conditions were examined:

1. VC: Fully vocoded simulation where vocoding is applied to the full frequency range between $80 \mathrm{~Hz}$ and $6000 \mathrm{~Hz}$ to simulate electric hearing only. This may be thought of as simulating a cochlear implant listener without residual low-frequency hearing.

2. VC + LP: Vocoded high frequencies above $661 \mathrm{~Hz}$ combined with low-pass filtered signal below $661 \mathrm{~Hz}$ which has undergone no processing in the low frequencies to simulate EAS. This may be thought of as simulating a cochlear implant listener with completely unimpaired residual low-frequency hearing.

3. $\mathbf{V C}+\mathbf{L P}(\mathbf{S M 3})$ : Vocoded high frequencies above $661 \mathrm{~Hz}$ combined with low-pass filtered signal below $661 \mathrm{~Hz}$ which has undergone spectral smearing with a smearing factor of $\mathrm{x} 3$ in the low frequencies to simulate EAS. This may be thought of as simulating a cochlear implant listener with residual low-frequency hearing with reduced frequency selectivity. 
4. VC + LP(SM6): Vocoded high frequencies above $661 \mathrm{~Hz}$ combined with low-pass filtered signal below $661 \mathrm{~Hz}$ which has undergone spectral smearing with a smearing factor of x6 in the low frequencies. This may be thought of as simulating a cochlear implant listener with residual low-frequency hearing with even poorer frequency selectivity.

5. $\mathbf{V C}+\mathbf{L P}(\mathbf{S M 3}+\mathbf{A 3 0})$ : Vocoded high frequencies above $661 \mathrm{~Hz}$ combined with lowpass filtered signal below $661 \mathrm{~Hz}$ which has undergone spectral smearing with a smearing factor of $\mathrm{x} 3$. Additionally $30 \mathrm{~dB}$ of attenuation has been applied to the low frequencies. This may be thought of as simulating a cochlear implant listener with residual low-frequency hearing with reduced frequency selectivity and $30 \mathrm{~dB}$ of lowfrequency threshold shift.

6. $\mathbf{V C}+\mathbf{L P}(\mathbf{S M 3}+\mathbf{A 6 0})$ : Vocoded high frequencies above $661 \mathrm{~Hz}$ combined with lowpass filtered signal below $661 \mathrm{~Hz}$ which has undergone spectral smearing with a smearing factor of $\mathrm{x} 3$. Additionally $60 \mathrm{~dB}$ of attenuation has been applied to the low frequencies. This may be thought of as simulating a cochlear implant listener with residual low-frequency hearing with reduced frequency selectivity and $60 \mathrm{~dB}$ of lowfrequency threshold shift.

\subsection{Test Conditions and Procedures}

Each participant completed three blocks of 32 sentences for each of the six signal processing conditions. Each block was presented at a different signal-to-noise ratio (SNR). The SNRs were selected for each condition to bracket the speech recognition threshold (SRT). The SRT is the SNR at which $50 \%$ of sentences are identified correctly. On a small number of occasions where the predetermined SNRs did not bracket the SRT for a participant, a block at an additional SNR was completed. Psychometric functions (recognition 
score as a function of SNR) were generated, from which the SRT was derived. The order of sentences within a list was randomized for each block; and the order of conditions was counterbalanced across participants.

During a block of trials, participants were presented with a range of coloured digits on a computer screen. Following the presentation of each sentence, participants were required to select the correct coloured digit. Therefore, for a trial to be scored as correct, the listener was required to accurately report both the colour and number. Participants were able to take breaks between blocks as needed.

Prior to testing, each participant completed a practice block of 10 unprocessed sentences presented at $0 \mathrm{~dB}$ SNR. Practice sentences were delivered by the same target speaker and with the same background speakers as used in the simulation conditions. The purpose of the practice block was to familiarize participants with the task as well as the target speaker's voice. This, combined with randomization of the order of conditions was felt to account for any anticipated learning effect.

Measurements were made in a single-walled sound-proof booth. Stimulus processing, data collection, and data analysis were controlled by custom software written in MATLAB, on an Acer Aspire 5738G Laptop with a Realtek HD audio soundcard, running Windows 8. Speech waveforms were processed in advance and saved for later use. For data collection, waveforms were read from files, and delivered monaurally to the right ear through circumaural headphones (Sennheiser HD280 Pro) at an overall level of $65 \mathrm{~dB}$ SPL. A Tektronix TDS 2002 two channel digital storage oscilloscope was used as a voltmeter to measure the voltage output from the soundcard in order to determine the gain setting of the soundcard which would result in an output of $65 \mathrm{~dB}$ SPL. Once collected, data was analysed using IBM SPSS software. Significance level was set at $\mathrm{p}<0.05$. 


\section{Results}

\subsection{Psychometric functions}

The speech recognition scores for each subject at varying SNRs were used to determine the psychometric function of each condition. A representative psychometric function for an individual subject is displayed in Figure 7. The dark blue lines in Figure 7 each represent one of the six listening conditions (C1- C6). Refer to section 7.4 for more information regarding the signal processing involved for each condition. The horizontal light blue line in Figure 7 represents the 50\% speech recognition score. The point at which this line meets the psychometric function of each condition represents the SRT of that condition. In other words, the SRT of each condition was the SNR at which the speech recognition score was $50 \%$ correct. In Figure 7, this was $2 \mathrm{~dB}$ SNR for the VC condition (C1), $-1.7 \mathrm{~dB}$ SNR for the VC + LP condition (C2), -1.6 dB SNR for the VC + LP(SM3) condition (C3), -1.6 dB SNR for the VC + LP(SM6) condition (C4), 0.8 dB SNR for the VC + LP(SM3+A30) condition (C5), and 4.5 dB SNR for the VC + LP(SM3+A60) condition (C6). 


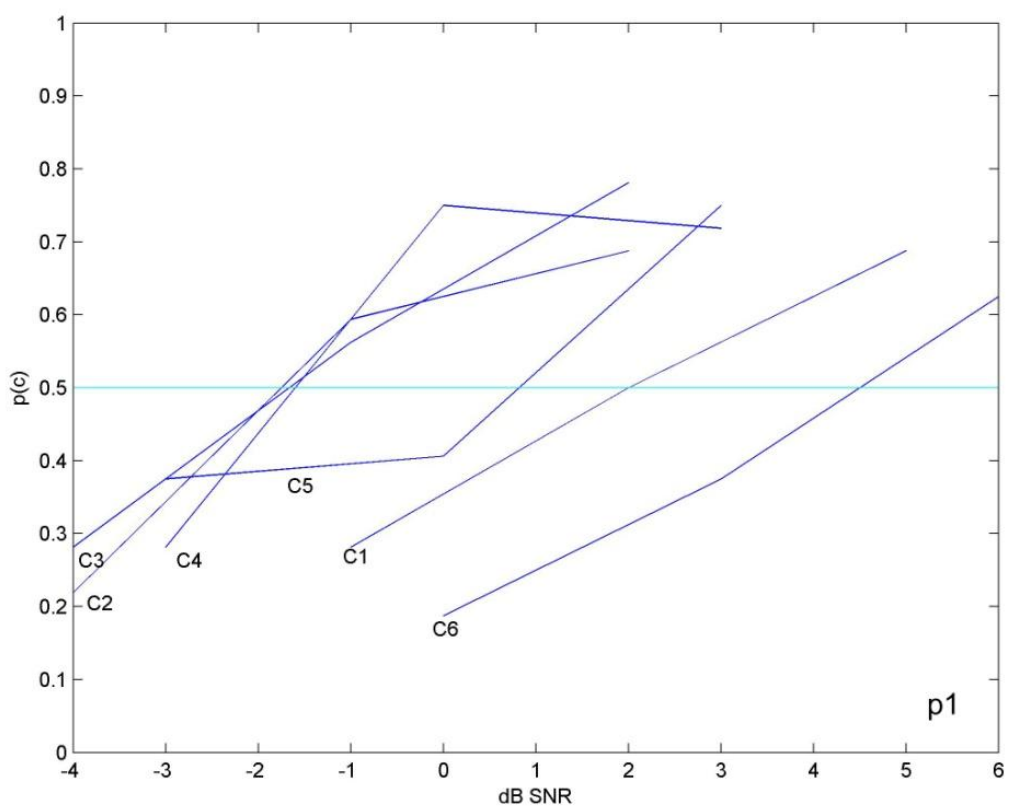

Figure 7: Psychometric function of speech recognition score as a function of SNR for Participant 1

Further representative psychometric functions can be seen in Figure 8 and Figure 9. The SRTs for each condition can be obtained from these figures in the same way as described for Figure 7. Overall, variation in the shape of the psychometric functions can be observed between conditions and individuals, but the general trend, in which a higher signal to noise ratio results in a higher speech recognition score, is illustrated in most cases. 


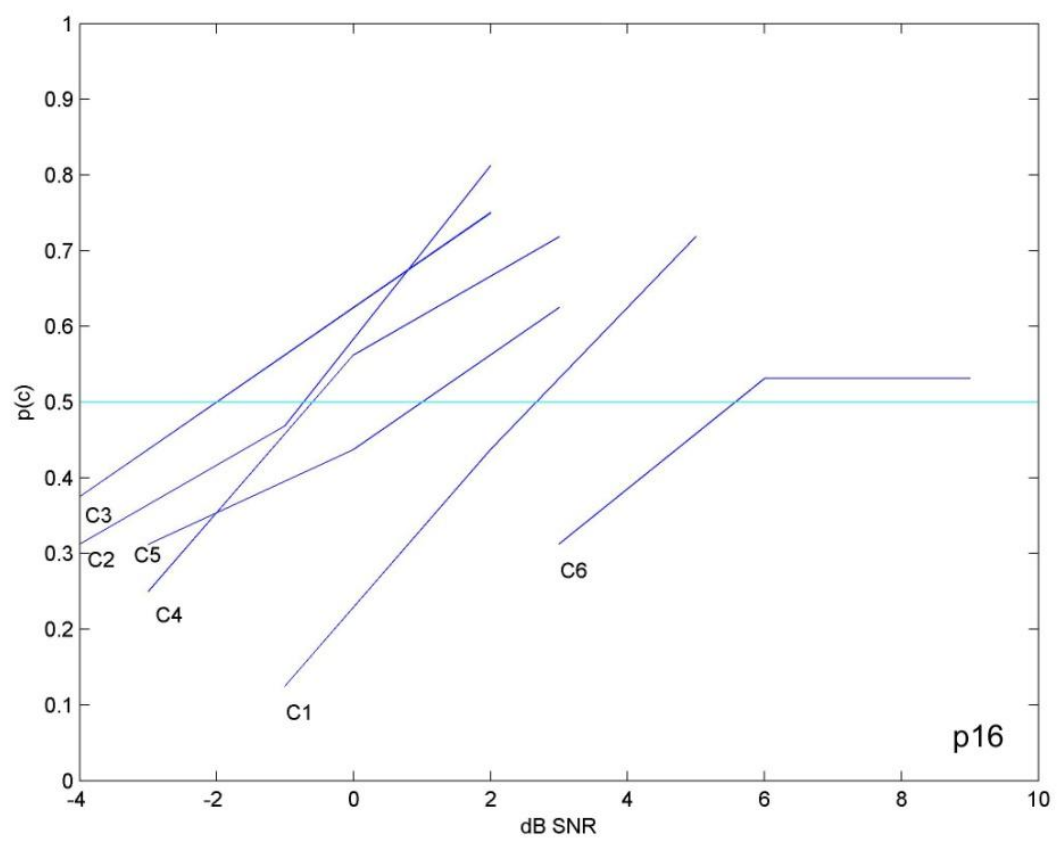

Figure 8: Psychometric function of speech recognition score as a function of SNR for Participant 16

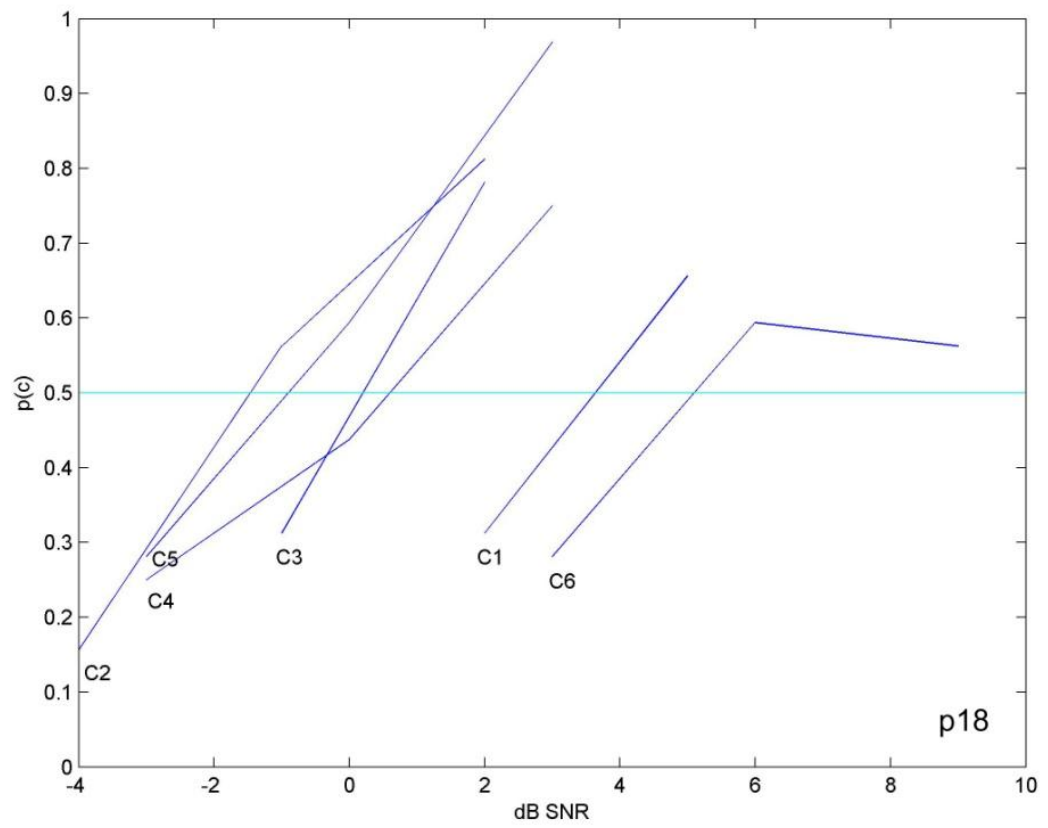

Figure 9: Psychometric function of speech recognition score as a function of SNR for Participant 18 


\subsection{Statistical analysis and findings}

The box plot in Figure 10 shows the median SRT, in dB SNR, for each listening condition. The upper edge of each box represents the upper quartile of the data while the lower edge of each box represents the lower quartile of the data. Also displayed by the upper and lower whiskers of each box are the maximum and minimum values of the data.

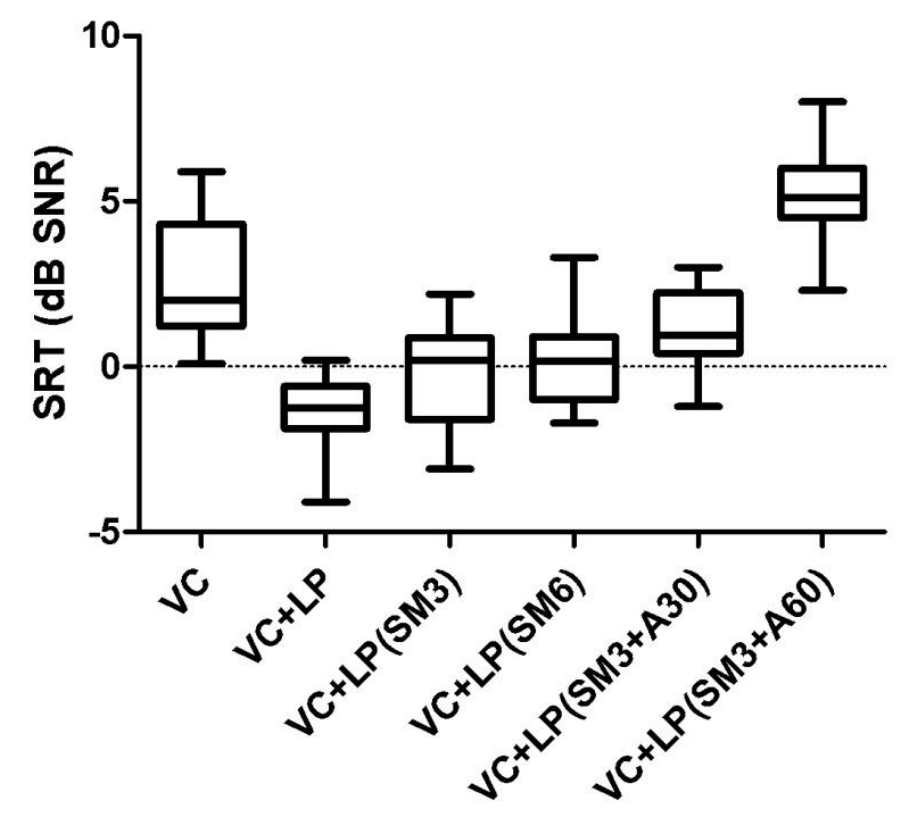

Figure 10: Box Plot of SRT for each condition

A one-way repeated measures ANOVA was conducted in IBM SPSS software, with condition as the within subject variable. Significance level was set at $p \leq 0.05$. Results from the one-way Repeated Measures ANOVA conducted on the SRT values revealed a significant condition effect $[F(5,70)=62.15, p<0.001]$. Mauchly's Test of Sphericity was nonsignificant indicating that the variances of the differences are roughly equal and therefore the assumption of sphericity was not violated. 
Pairwise comparisons revealed that the SRT for the VC + LP(SM3+A60) condition was significantly higher than the SRT in any of the other conditions $(\mathrm{M}=5.3, \mathrm{SD}=1.5)$. A higher SRT generally implies a condition more detrimental to speech perception. This was followed by the $\mathrm{VC}$ condition $(\mathrm{M}=2.3, \mathrm{SD}=1.7)$, which had a significantly higher SNR SRT than the VC $+\mathrm{LP}$ condition $(\mathrm{M}=-1.6, \mathrm{SD}=1.1)$, the $\mathrm{VC}+\mathrm{LP}(\mathrm{SM} 3)$ condition $(\mathrm{M}=-$ $0.6, \mathrm{SD}=1.5)$, and the $\mathrm{VC}+\mathrm{LP}(\mathrm{SM} 6)$ condition $(\mathrm{M}=-0.1, \mathrm{SD}=1.3)$, but revealed no significant difference to the $\mathrm{VC}+\mathrm{LP}(\mathrm{SM} 3+\mathrm{A} 30)$ condition $(\mathrm{M}=1, \mathrm{SD}=1.2)$. The $\mathrm{SNR}$ SRT of the VC + LP(SM3+A30) condition was shown to be significantly lower than that of the $\mathrm{VC}+\mathrm{LP}(\mathrm{SM} 3+\mathrm{A} 60)$ condition and significantly higher than that of the VC + LP condition, but showed no significant difference to any of the other conditions. There was no significant difference between conditions VC + LP, VC + LP(SM3), and VC + LP(SM6).

The benefit for speech in noise of simulated EAS over simulated electric hearing alone can be visualized in Figure 11. The SRT for each of the EAS conditions is shown in $\mathrm{dB}$ relative to the SRT for the VC condition. In the current study, this difference is referred to as EAS Benefit $(\mathrm{dB})$. In cases where the EAS conditions fall above the $0 \mathrm{~dB}$ line in Figure 11, an advantage in SRT (dB SNR) over the VC condition is indicated. 


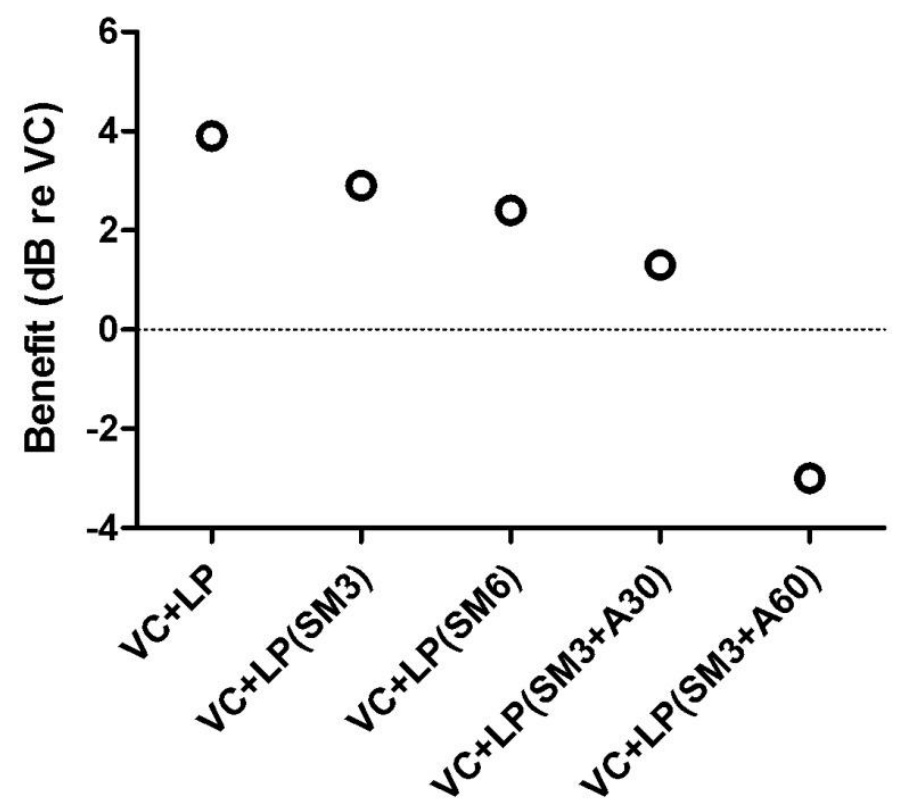

\section{Figure 11: Benefit (dB) of EAS listening conditions compared to VC condition}

The EAS benefit was $3.9 \mathrm{~dB}$ when low frequency sound was unprocessed, $2.9 \mathrm{~dB}$ when low frequency sound had spectral smearing of x3 applied, $2.4 \mathrm{~dB}$ when low frequency sound had spectral smearing of x6 applied. When $30 \mathrm{~dB}$ attenuation was applied as well as x3 spectral smearing, no significant benefit was observed. When $60 \mathrm{~dB}$ attenuation was applied as well as $\mathrm{x} 3$ spectral smearing, a significant negative relationship was found, with a $3 \mathrm{~dB}$ disadvantage for simulated EAS listening with this degree of simulated hearing loss and reduced frequency selectivity in the low frequencies compared to simulated electric stimulation alone.

Differences between the three conditions in which no attenuation was applied to the low frequency region and condition VC can be visualized in figure 12. The EAS benefit is demonstrated in this figure, with each of the EAS conditions having significantly improved SRTs compared to the VC condition. 


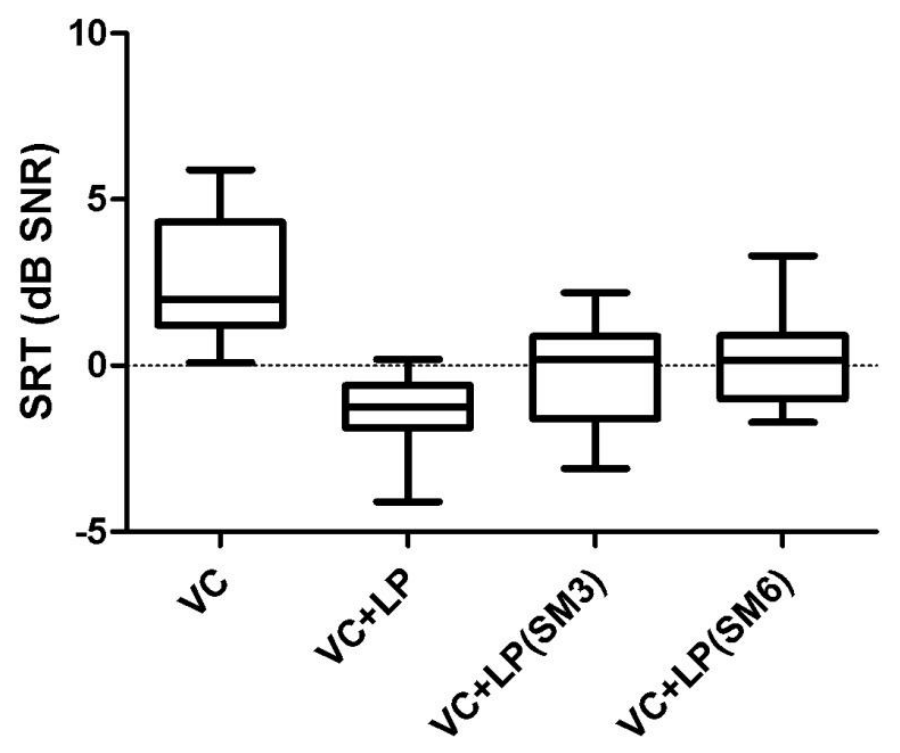

\section{Figure 12: SRT (dB SNR) of the VC condition and three EAS conditions with no attenuation applied to the low frequency region}

The difference between the EAS conditions in which only spectral smearing was applied to the low frequency region and the EAS conditions in which both spectral smearing and attenuation was applied to the low frequency region may be said to simulate the effect of providing amplification to the low frequency region, as will be discussed further in section 9.1.2. This improvement in SRT representing the simulated difference between EAS listening with and without amplification of the low frequency region can be visualized in Figure 13. This figure allows comparisons between the VC $+\mathrm{LP}(\mathrm{SM} 3)$ condition and the VC + $\mathrm{LP}(\mathrm{SM} 3+\mathrm{A} 30)$ and $\mathrm{VC}+\mathrm{LP}(\mathrm{SM} 3+\mathrm{A} 60)$ conditions, all of which have the same amount of spectral smearing, but different degrees of attenuation applied to the low frequency region. 


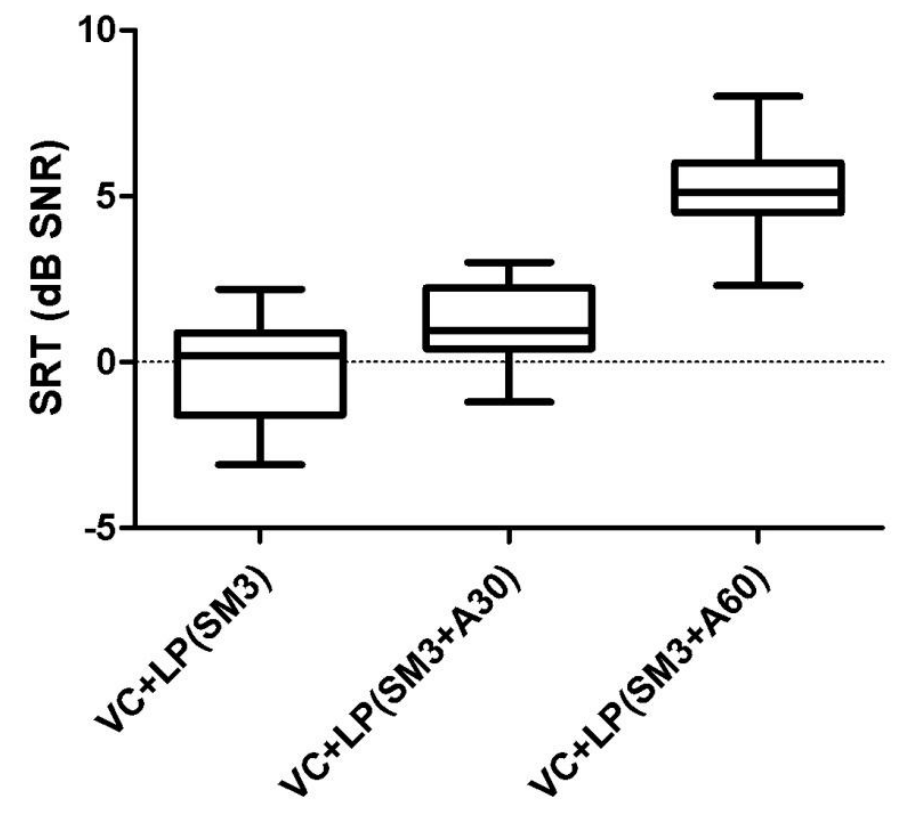

Figure 13: SRT (dB SNR) of EAS conditions with $x 3$ spectral smearing and varying degrees of attenuation applied to the low frequency region

SRTs for all individual participants can be found in Table 2.

Table 2: Individual SRTs for each condition

\begin{tabular}{|c|c|c|c|c|c|c|}
\hline $\begin{array}{l}\text { Participant } \\
\text { number }\end{array}$ & $\begin{array}{l}\text { VC SRT } \\
\text { (dB SNR) }\end{array}$ & $\begin{array}{l}\text { VC+LP SRT } \\
\text { (dB SNR) }\end{array}$ & $\begin{array}{l}\mathrm{VC}+\mathrm{LP} \\
\text { (SM3) SRT } \\
\text { (dB SNR) }\end{array}$ & $\begin{array}{l}\text { VC+LP } \\
(\mathrm{SM} 6) \text { SRT } \\
(\mathrm{dB} \text { SNR) }\end{array}$ & $\begin{array}{l}\mathrm{VC}+\mathrm{LP} \\
(\mathrm{SM} 3+\mathrm{A} 30) \\
\text { SRT } \\
\text { (dB SNR) }\end{array}$ & $\begin{array}{l}\mathrm{VC}+\mathrm{LP} \\
(\mathrm{SM} 3+\mathrm{A} 60) \\
\text { SRT } \\
(\mathrm{dB} \text { SNR) }\end{array}$ \\
\hline 1 & 2 & -1.8 & -1.7 & -1.6 & 0.8 & 4.5 \\
\hline 2 & 0.8 & -4.1 & -1.3 & 0.6 & 0.4 & 2.3 \\
\hline 3 & 0.8 & -1 & -0.1 & 3.3 & 2.5 & 6 \\
\hline 4 & 4.5 & -1.5 & 0.2 & 1.6 & 2.4 & $>7$ (excluded) \\
\hline 5 & 3.5 & -0.1 & 0.5 & -1.1 & -0.5 & $>7$ (excluded) \\
\hline 6 & 5.4 & -1.6 & 0.2 & -1 & 1.7 & 5 \\
\hline 7 & 2 & -0.6 & -2.1 & -1.7 & 0.3 & 3.7 \\
\hline 8 & 1.5 & -3 & -0.1 & 0 & 1.3 & 4.5 \\
\hline 9 & 1.2 & -1.9 & -1.9 & -0.3 & 3 & 7.3 \\
\hline 10 & 4.1 & -0.1 & 0.2 & -0.8 & 3 & 8 \\
\hline 11 & 2 & -1 & 0.8 & 0.3 & 0.5 & $>9$ (excluded) \\
\hline 12 & 5.9 & -0.2 & 0.9 & 0.6 & 0.4 & 7.3 \\
\hline 13 & 5.2 & -0.8 & 1.5 & 2.1 & 1.6 & $>9$ (excluded) \\
\hline 14 & 2 & -1.7 & 2.2 & -1 & 0.9 & 4.5 \\
\hline 15 & 0.1 & -2.5 & -3.1 & 0.5 & 1 & 6 \\
\hline 16 & 2.7 & -0.7 & -2 & -0.6 & 1 & 5.6 \\
\hline 17 & 4.4 & 0.2 & 1.5 & 1 & 3 & $>12$ (excluded) \\
\hline 18 & 3.6 & -1.5 & 0.2 & 0.6 & -0.9 & 5.1 \\
\hline 19 & 1.3 & -0.6 & 1.2 & 1 & 0.9 & 6 \\
\hline 20 & 0.9 & -2.4 & -1.3 & -1 & -1.2 & 4.1 \\
\hline
\end{tabular}




\subsection{Excluded Data}

Out of our twenty participants, we did not obtain an SRT from five participants for one condition due to not having waveforms at high enough SNRs generated. Five of our participants did not reach a recognition score of $50 \%$ or above for the $\mathrm{VC}+\mathrm{LP}(\mathrm{SM} 3+\mathrm{A} 60)$ condition, even at the highest SNRs presented (12 dB SNR). These results were excluded from the formal data analysis described above.

Interestingly, for four out of five of these participants, condition VC + LP(SM3+A60) was presented either first or second in the order of testing. At the end of the experiment, three of these participants were presented with condition $\mathrm{VC}+\mathrm{LP}(\mathrm{SM} 3+\mathrm{A} 60)$ at the same SNRs as they were presented with at the start of the experiment. All three participants were able to obtain speech recognition scores of $>50 \%$ when re-presented with condition VC $+\mathrm{LP}(\mathrm{SM} 3+\mathrm{A} 60)$ at the end of the experiment. This indicates a learning effect, which seemed to be particularly evident for this condition.

The PRE- and POST psychometric functions representing scores at the start of the experiment compared to the end of the experiment for condition VC $+\mathrm{LP}(\mathrm{SM} 3+\mathrm{A} 60)$ for these three participants are shown in Figure 14, Figure 15, and Figure 16. In the following figures upward movement of the line indicates that a larger percentage of speech was recognized correctly. If the line moves to the left it indicates speech was recognized at lower signal to noise ratios. Figures 14, 15, and 16 all demonstrated an upwards movement, a movement to the left, or both. 


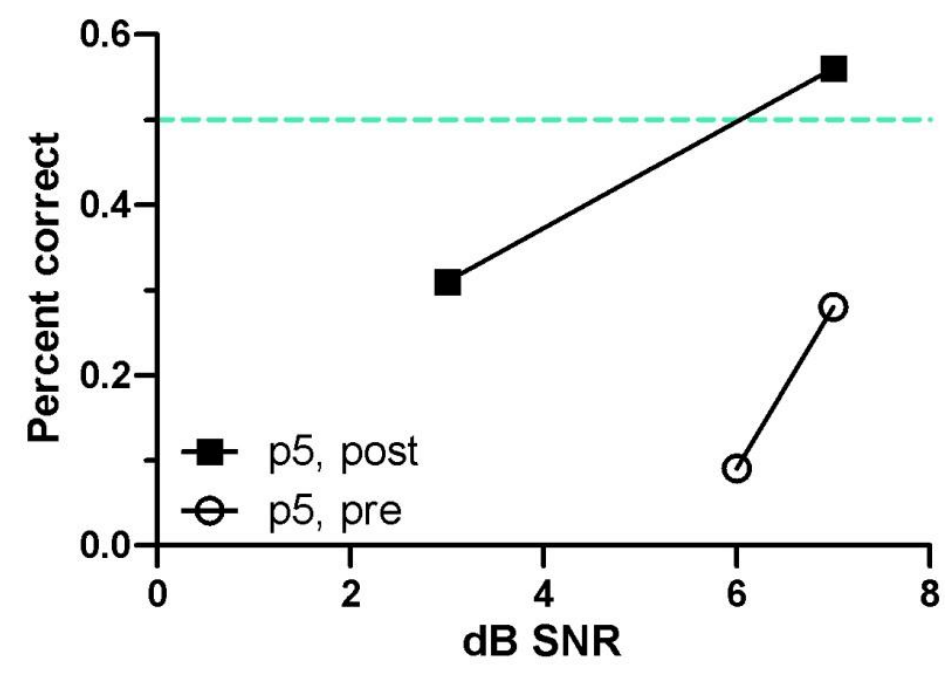

Figure 14: Participant 5 PRE- and POST psychometric functions illustrating speech perception scores as a function of $\mathrm{dB}$ SNR at the start of the experiment and at the end of the experiment for Condition 6

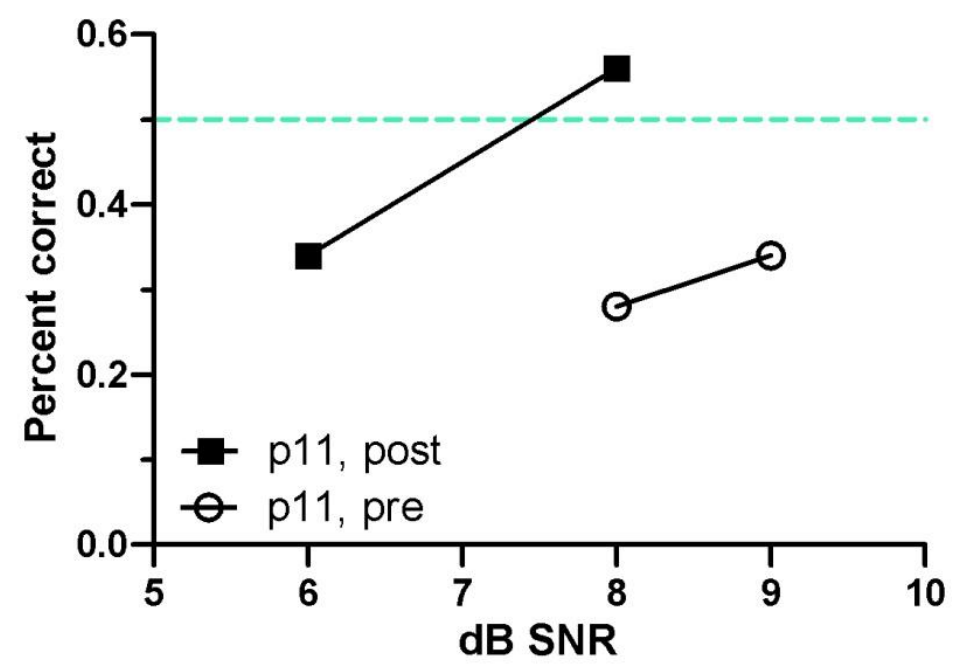

Figure 15: Same as Fig. 14, for Participant 11 


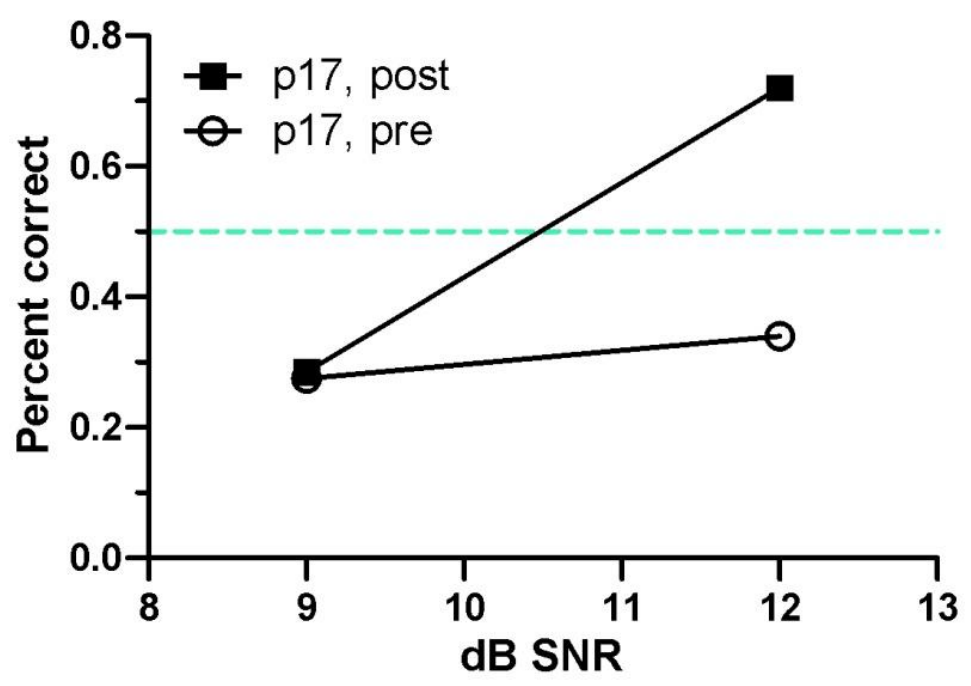

Figure 16: Same as Fig. 14, for Participant 17 


\section{Discussion}

\subsection{General Discussion}

The aim of this study was to examine the effects of spectral smearing and attenuation of low frequency information on the identification of vocoded speech in noise. This was done in order to investigate the benefit for speech recognition in background noise that is commonly reported for combined EAS hearing. Simulation of EAS was achieved by combining vocoded high frequency sound with low frequency sound. Many EAS listeners experience reduced frequency selectivity and elevated audiometric thresholds of their residual hearing as a consequence of sensorineural hearing loss. In order to simulate reduced frequency selectivity and elevated audiometric thresholds, varying degrees of spectral smearing and attenuation were applied to the low frequency region. This chapter discusses the findings of this study, how it relates to the hypotheses, and how it compares to previous literature in this field. Limitations of the current study and directions for future research will also be discussed.

\subsubsection{Hypothesis 1}

We hypothesized that spectral smearing and attenuation of low frequency information would have a detrimental effect on the recognition of vocoded speech in noise. It was also anticipated that signals with a larger degree of spectral smearing and attenuation would require a higher signal-to-noise ratio (SNR) to maintain intelligibility. In regards to spectral smearing, this hypothesis was formulated on the bases of previous literature which has shown that scores for speech recognition in noise tend to decrease with increasing auditory filter width as well as with decreasing SNR (Baer \& Moore, 1993, 1994). The hypothesised detrimental effect of attenuation was founded on the clinical experience of patients with reduced hearing thresholds and their reported difficulties with speech in noise. 
In our study, the VC + LP condition was a speech signal with vocoded high frequencies and no processing of the low frequencies. In Conditions VC + LP(SM3), VC + $\mathrm{LP}(\mathrm{SM} 6), \mathrm{VC}+\mathrm{LP}(\mathrm{SM} 3+\mathrm{A} 30)$, and $\mathrm{VC}+\mathrm{LP}(\mathrm{SM} 3+\mathrm{A} 60)$ the low frequency band below $661 \mathrm{~Hz}$ was processed to have increasing amounts of spectral smearing, attenuation, or both. As seen in Figure 10, the SRT increased as increasing amounts of spectral smearing and attenuation were applied to the low frequency information of the speech signal. The increase in SNR resulting from spectral smearing and attenuation was not significant for all conditions however. The difference between conditions VC + LP, VC + LP(SM3), and VC + LP(SM6) was found to be non-significant, suggesting spectral smearing in isolation was not significantly different from no processing, even when smearing was applied to simulate auditory filter widths $6 \mathrm{x}$ broader than normal .

This was a surprising finding as it is in contrast to findings from previous literature (Baer \& Moore, 1993, 1994), which showed that speech recognition performance in both speech-shaped noise and interfering speech was reduced when broadening of auditory filters was simulated. The method of spectral smearing used in the current study was adapted from Baer \& Moore, (1993), and matched it as closely as possible. The same degrees of auditory filter width broadening ( 33 and $x 6$ ) were simulated in both studies. Perhaps the reason our results showed no significant effect of low frequency spectral smearing for speech in noise recognition is because the detrimental effect of spectral smearing might have been negligible in the presence of vocoding of the high frequencies, which was likely to have a much larger impact on speech intelligibility. It was only when spectral smearing was combined with attenuation of the low frequencies that a significant detrimental effect on speech recognition scores in background noise was noted. This is evidenced by the fact that speech recognition scores for $\mathrm{VC}+\mathrm{LP}(\mathrm{SM} 3+\mathrm{A} 30)$ and $\mathrm{VC}+\mathrm{LP}(\mathrm{SM} 3+\mathrm{A} 60)$ conditions, both of which had spectral smearing as well as attenuation of the low frequencies, were significantly poorer than 
the speech recognition scores for the VC + LP condition, in which low frequency sound was unprocessed.

\subsubsection{Hypothesis 2}

Furthermore, we anticipated significant benefit for speech recognition in noise when combining simulated acoustic and electric hearing, compared to simulated electric hearing alone. We hypothesised that this benefit would be evident despite spectral smearing and attenuation of the low frequencies. Results for VC + LP, VC + LP(SM3) and VC + LP(SM6) conditions followed this expected trend with significant benefit observed for simulated EAS compared to electric stimulation only for speech in noise. The EAS benefit was $3.9 \mathrm{~dB}$ when low frequency sound was unprocessed, $2.9 \mathrm{~dB}$ when low frequency sound had spectral smearing of $\mathrm{x} 3$ applied, and $2.4 \mathrm{~dB}$ when low frequency sound had spectral smearing of x6 applied as seen in Figure 12. These results were promising as it indicated that even with a large reduction in frequency selectivity of the auditory system, as occurs with broadened auditory filters, EAS recipients are likely to still benefit from the combined mode of stimulation.

This hypothesis did not hold true for all the EAS conditions in our experiment however. The VC $+\mathrm{LP}(\mathrm{SM} 3+\mathrm{A} 60)$ condition, in which a smearing factor of $\mathrm{x} 3$ as well as 60 $\mathrm{dB}$ attenuation had been applied to the low frequency information, had a significantly higher SRT than the VC condition, which simulated electric hearing only. Bearing in mind these results are based on simulations and not actual EAS performance, the results seem to indicate that individuals with moderately elevated thresholds in the low frequencies as well as auditory filters broader than normal, might benefit more from a traditional full cochlear implant than a partially inserted hybrid device. Not only did this degree of simulated hearing loss and reduced frequency selectivity in the low frequencies result in a significantly higher 
SRT than all other conditions; overall participants also found this condition to be very challenging.

The results of this study also found that there was no significant difference between the VC condition, which simulated electric hearing without residual low-frequency hearing, and the $\mathrm{VC}+\mathrm{LP}(\mathrm{SM} 3+\mathrm{A} 30)$ condition, which simulated EAS stimulation with $\mathrm{x} 3$ spectral smearing and $30 \mathrm{~dB}$ attenuation applied to the low frequencies. The SRT of the VC condition was $1.3 \mathrm{~dB}$ SNR higher than the SRT of the $\mathrm{VC}+\mathrm{LP}(\mathrm{SM} 3+\mathrm{A} 30)$ condition. Even though 1.3 dB SNR may be enough to make a functional difference to speech recognition in everyday life, we are unable to attribute this difference to anything more than chance based on our results. The clinical application of these results would indicate that an individual with severe to profound high frequency hearing loss and a mild hearing loss combined with auditory filters broader than normal in the low frequencies may not receive additional benefit from combined EAS as compared to complete electric stimulation alone. However, it is important to bear in mind the limitations of simulation studies, as outlined in section 4.5 and later in this review, when making such inferences.

It was a somewhat surprising finding that two of the EAS conditions, $\mathrm{VC}+$ LP(SM3+A30) and VC + LP(SM3+A60), did not have significantly improved SRTs compared to the VC condition. This is because large benefit has been reported in studies with actual EAS users for combined EAS listening compared to electric stimulation alone, even when participants presented with a moderately-severe to severe degree of hearing loss in the residual low frequencies. Kiefer et al. (2005) described the case of a patient who, despite a moderately-severe hearing loss in the low frequencies and poor pre-operative monosyllabic word scores, was able to obtain a 52\% increase in speech in noise scores for combined EAS compared to cochlear implant listening with no low frequency signal. Luetje et al. (2007) reported significantly better SRTs with combined EAS for two patients with a severe degree 
of hearing loss in the low frequencies. Kong et al. (2005) demonstrated that even when residual hearing is severely impaired and insufficient for speech recognition, the remaining signal can still contribute to the EAS benefit over electric-alone hearing. In a clinical trial by Turner et al. (2008) a regression line drawn through data points representing the SRT as a function of residual low frequency thresholds suggested that the EAS benefit is likely to be present until residual hearing approaches a profound degree of hearing loss.

One of the likely explanations for the lack of EAS benefit when elevated thresholds in low frequency hearing was simulated in our study is that this manipulation does not account for the effect of providing amplification in the low frequency region. A majority of clinical trials report the use of additional amplification to increase audibility of low frequency sound in cases of reduced hearing thresholds in this region (Gantz et al., 2005; Gantz et al., 2006; Gstoettner et al., 2009; Kiefer et al., 2004; Kiefer et al., 2005; Kong et al., 2005; Luetje et al., 2007; Turner et al., 2004). However, details regarding the use of this amplification such as how many participants received amplification, what hearing loss was considered appropriate for additional amplification, whether it was provided through a separate hearing aid device or through a hybrid cochlear implant device, and what fitting procedures were used, was usually not clearly specified. In a number of studies involving actual EAS users it was unclear or unspecified whether additional amplification was provided for the low frequency region (Dorman et al., 2008; Lorens et al., 2008; Turner et al., 2008; Turner et al., 2010). One of the studies reviewed explicitly said that no additional amplification was provided in the low frequency region due to the participant having near normal low frequency hearing thresholds (20 - $25 \mathrm{~dB}$ HL at $500 \mathrm{~Hz}$ and below) (Turner et al., 2004). In another study by Helbig et al. (2008) all 9 participants were reported to initially wear a separate in-the-ear (ITE) hearing aid in the implanted ear, but 6 participants were reported to subsequently stop wearing their ITE 
hearing aids due to the inconvenience of having to manage two separate devices, or feeling that the additional amplification did not provide extra benefit.

Looking at previous literature we may assume that in most EAS studies, the participant was likely to also receive amplification through traditional hearing aid technology to increase the audibility of low frequency sounds. While this type of amplification won't correct for the effects of broadened auditory filters, and therefore speech intelligibility, it is at least likely to make speech more audible.

It may be argued that the $\mathrm{VC}+\mathrm{LP}(\mathrm{SM} 3)$ and $\mathrm{VC}+\mathrm{LP}(\mathrm{SM} 6)$ conditions in the current study simulate the effect of providing amplification to the low frequency region in EAS listening. As simulated by these conditions, amplification in the low frequency region would account for elevated thresholds, but the effects of broadened auditory filters would still be present. Since conditions VC + LP(SM3) and VC + LP(SM6) showed significant benefit over the VC condition, it may be inferred that the simulated effect of providing amplification in the low frequency region results in significant EAS benefit. To look at the simulated effect of not providing amplification in the low frequency region, we might consider conditions VC + LP(SM3), VC + LP(SM3+A30), and VC + LP(SM3+A60) all of which have x3 spectral smearing applied in the low frequencies. In addition to spectral smearing, the $\mathrm{VC}+$ $\mathrm{LP}(\mathrm{SM} 3+\mathrm{A} 30)$ and $\mathrm{VC}+\mathrm{LP}(\mathrm{SM} 3+\mathrm{A} 60)$ conditions also have attenuation of the low frequency sound, with $30 \mathrm{~dB}$ attenuation for condition $\mathrm{VC}+\mathrm{LP}(\mathrm{SM} 3+\mathrm{A} 30)$, and $60 \mathrm{~dB}$ attenuation for condition $\mathrm{VC}+\mathrm{LP}(\mathrm{SM} 3+\mathrm{A} 60)$. We may therefore assume that the difference between the $\mathrm{VC}+\mathrm{LP}(\mathrm{SM} 3)$ condition and the $\mathrm{VC}+\mathrm{LP}(\mathrm{SM} 3+\mathrm{A} 30)$ and $\mathrm{VC}+$ LP(SM3+A60) conditions might indicate the difference between having an appropriately fit hearing aid that makes sounds audible in the low frequencies compared to not using amplification in the low frequencies. The improvement in SRT that represents the simulated difference between EAS listening with and without amplification of the low frequency region 
can be visualized in Figure 13. It is believed these results thus support the use of amplification in the low frequencies for EAS recipients with reduced hearing thresholds in this frequency range.

\subsection{Comparisons of EAS benefit with previous literature}

It is possible to draw comparisons between the results of the current study and those of previous EAS simulation studies. In drawing these comparisons we must consider the VC condition, which simulated electric stimulation only, and the VC + LP condition, which simulated EAS listening with no processing of the low frequency acoustic information, as these conditions are most similar to those described in previous simulation studies. In their study, Turner et al. (2004) reported much lower SRT levels for both their simulated electric only and simulated EAS conditions than was found in our study. This could be due to a range of factors including the use of spondee words as the target speech compared to sentences in the current study, and the use of a 16-channel simulation compared to 8-channel simulation used in our experiment. Despite this, the advantage of simulated EAS over simulated electric only stimulation for speech in noise was similar between the two studies. Turner et al. (2004) reported an advantage of $4.9 \mathrm{~dB}$ for simulated EAS over electric stimulation only, while our results indicated an advantage of $3.9 \mathrm{~dB}$. Results even more similar to ours were reported by Li and Loizou (2008). They found that for speech in the background of a single competing talker the SRT for the simulated electric only condition was $3 \mathrm{~dB}$ SNR compared to $-2.5 \mathrm{~dB}$ SNR for the simulated combined EAS condition. The resulting benefit was thus $5.5 \mathrm{~dB}$. Their estimated electric only condition SRT was approximately $0.7 \mathrm{~dB}$ higher than ours and their estimated EAS condition SRT was about $0.9 \mathrm{~dB}$ lower than ours. Our results were also similar to that of Qin and Oxenham (2006) who reported a SRT of approximately 3.5dB SNR for the simulated electric only condition and an SRT of approximately $-2.5 \mathrm{~dB}$ SNR for the simulated EAS condition for sentences in noise with a competing talker background. 
Overall there are many similarities between the results of our study and those of previous simulation studies investigating EAS benefit in noise. Our main interest, however, lies in comparing our results to those of studies in which actual EAS users were recruited to investigate EAS benefit in noise. As stated by Qin \& Oxenham (2006, p. 2424): "However, it is important to note that our simulations involve "ideal" residual hearing, with no hearing loss and accompanying effects, such as broadened auditory filters. These conditions are unlikely to hold in real EAS users.”. Li and Loizou (2008), and Yang and Zeng (2013) also identified this assumption of 'ideal' low frequency hearing as a limitation in EAS simulation studies. Since the goal of the methodology of this study was to simulate low frequency hearing more similar to that experienced by real EAS recipients, we are even more interested in comparing our results to those of studies with real EAS users as participants. This holds especially true for the VC + LP(SM3), VC + LP(SM6), VC + LP(SM3+A30), and VC + LP(SM3+A60) conditions; all of which simulate varying degrees of low frequency hearing loss and broadening of auditory filters.

Table 3 compares data reported by Turner et al. (2004), Gantz et al. (2005), and Gantz et al. (2006) for participants in the clinical trial of the Iowa/Nucleus Hybrid cochlear implant with data obtained in the current simulation study. For each of the clinical trial studies, the SRT for speech in a background noise of competing talkers is reported for a group of recipients of the Iowa/Nucleus hybrid cochlear implants who were receiving combined EAS, as well as for a group of matched traditional long-electrode cochlear implant users. For the current simulation study, the SRT for the VC condition, which simulates traditional longelectrode cochlear implant processing, was reported as the electric stimulation only value. The SRTs of conditions VC + LP, VC + LP(SM3), VC + LP(SM6), VC + LP(SM3+A30), and $\mathrm{VC}+\mathrm{LP}(\mathrm{SM} 3+\mathrm{A} 60)$, which simulated combined EAS hearing with varying degrees of elevated hearing thresholds and reduced frequency selectivity in the low frequencies, were 
reported as the combined EAS values. The difference when the SRT for the combined EAS values are subtracted from the electric stimulation alone values have been reported as the EAS benefit (dB). This benefit refers to the improvement in SRT for combined EAS compared to full electric stimulation alone.

Table 3: Comparing EAS benefit between a group of clinical trials and the current study

\begin{tabular}{|c|c|c|c|}
\hline & $\begin{array}{l}\text { Full Electric Stimulation } \\
\text { SRT (dB SNR) }\end{array}$ & EAS SRT (dB SNR) & EAS Benefit (dB) \\
\hline Turner et al. (2004) & $7^{\star}$ & -7 & 14 \\
\hline Gantz et al. (2005) & $6.5^{\star}$ & -2 & 9 \\
\hline Gantz et al. (2006) & $8^{*}$ & -3 & 11 \\
\hline Current study & 2.3 & {$[\mathrm{VC}+\mathrm{LP}]$} & 3.9 \\
\hline Current study & 2.3 & {$[\mathrm{VC}+\mathrm{LP}(\mathrm{SM} 3)]$} & 2.9 \\
\hline Current study & 2.3 & {$[\mathrm{VC}+\mathrm{LP}(\mathrm{SM}$ 6)] } & 2.4 \\
\hline Current study & 2.3 & {$[\mathrm{VC}+\mathrm{LP}(\mathrm{SM} 3+\mathrm{A} 30)]$} & 1.3 \\
\hline Current study & 2.3 & {$[\mathrm{VC}+\mathrm{LP}(\mathrm{SM} 3+\mathrm{A} 60)]$} & -3 \\
\hline
\end{tabular}

As seen in Table 3 when comparing our results with those reported for the subjects who participated in the multicenter clinical trial of the Iowa/Nucleus Hybrid 10-mm cochlear implant, it would appear that our data underestimates the benefit of combined EAS for speech in noise. In order to shed light on one probable reason for this it is important to note the differences in SRT for the electric stimulation alone as seen in the left-hand row of Table 3. In the clinical trials studies, these SRTs were obtained from traditional long-electrode cochlear implant users. In our study the SRT was obtained from normal hearing listeners listening to the VC condition, which aimed to simulate traditional long-electrode cochlear implant processing using vocoding. It is evident that the SRT of the real cochlear implant users was much higher than the SRT of the simulated cochlear implant processing in the current study. These differences may be attributed to the limitations of using vocoding as a method of simulating cochlear implant processing, as will be discussed in further detail shortly. Because the SRT of the simulated cochlear implant processing in our study is 
comparatively low, the resulting benefit from the addition of low-pass filtered speech to simulate residual low frequency hearing was also comparatively reduced. We may be able to conclude, therefore, that one of the reasons our results show less of an EAS benefit is because our study overestimates the performance of traditional long-electrode cochlear implants.

\subsection{Limitations and Directions for Future Research}

One important consideration is that vocoding approximates the performance of the most successful cochlear implant users with the highest scores (Fishman et al. 1997; Friesen et al., 2001). This means that vocoding does not represent average cochlear implant performance for the general population, such as the groups of cochlear implant users who participated in the clinical trials outlined in Table 3. Cullington and Zeng (2008) measured speech recognition in normal-hearing, cochlear implant, and implant simulation subjects for varying background noise signals. They found that the simulation subjects performed similarly to the cochlear implant subjects in a background of steady state noise, but performed better in a background of competing talkers. They conclude that the cochlear implant simulation used in their study may not be an accurate representation of cochlear implant users' performance in a background of competing talkers. In the current study we also employed a background of competing talkers, which may in part account for some of the discrepancies between the speech recognition scores for our cochlear implant simulation group, and the groups of cochlear implant recipients in the clinical trials discussed. It should be noted, however, that Cullington and Zeng (2008) employed a sine-carrier vocoded simulation, while the current study used a noise-carrier vocoded simulation.

When simulating cochlear implant processing in normal hearing listeners we are also eliminating the effect of complex extraneous variables that may influence individuals' outcomes following cochlear implantation. These may include factors such as: age at 
implantation, duration of hearing loss, aetiology of hearing loss, language exposure and linguistic experience, personal motivation, and many more. Being able to control for these extraneous variables may well improve the reliability of our results, but we are likely to compromise our validity, as results may not be accurately generalized to the cochlear implant user population. These factors may well account for the apparent overestimation of cochlear implant performance seen in our study.

Another important factor shown to influence results is subject experience as evidenced by the learning effect observed in our study. This learning effect, illustrated particularly well by the excluded data in Figures 14, 15, and 16 is consistent with previous literature. Whitmall et al. (2007) outlines this effect of subject training, pointing out that studies which allowed for extensive subject training and practice often reported relatively higher speech recognition scores than studies that didn't (Shannon et al. 1995; Dorman et al. 1997). In anticipation of this learning effect, the current study randomized the order in which conditions were presented, as well as having participants undergo a practice trial of 10 sentences in order to familiarize them with the task and the speaker's voice. Despite this, the learning effect was still clearly observed in this study, as seen in Figures 14, 15, and 16, particularly for participants who started with Condition 6, the most challenging condition.

A gap in current EAS literature has been identified in the lack of studies addressing the effect of providing low frequency amplification. This gap might provide direction for future research which could specifically investigate the effect of providing low frequency amplification on the benefit for speech in noise that is typical with EAS. The results of the current study seem to indicate that addressing elevated hearing thresholds in the low frequencies can be highly beneficial and conversely when no attempt is made to increase audibility of the low frequency region then the benefit for speech in noise with EAS may be reduced or absent. Due to the limitations of simulations studies as outlined in this review, this 
hypothesis remains unsubstantiated without evidence provided from studies with actual EAS recipients.

\subsection{Summary and conclusions}

Overall, our results indicate that there is indeed a significant improvement in speech recognition in a background of competing speakers with combined EAS compared to electric stimulation only. However, when elevated hearing thresholds were simulated for the residual low frequency hearing, we found that this benefit was either absent or reversed. These results are consistent with the use of amplification for individuals with reduced hearing thresholds in the low frequencies in order to utilize the benefit they are able to achieve with combined EAS. If some degree of residual low frequency hearing can be preserved following hybrid cochlear implantation, and reduced thresholds in this region can be amplified to increase audibility, it is believed improved speech recognition scores in background noise may be achieved compared to traditional long-electrode cochlear implantation. 


\section{References}

Allen, J. B. (1977). Short term spectral analysis, synthesis and modification by discrete Fourier transform. IEEE Transactions on Acoustics, Speech and Signal Processing, 25(3), 235-238. DOI:10.1109/TASSP.1977.1162950

American Speech-Language-Hearing Association. (2004). Cochlear Implants [Technical Report]. Available from www.asha.org/policy. DOI:10.1044/policy.TR2004-00041

Arehart, K. H., Souza, P. E., Muralimanohar, R. K., \& Miller, C. W. (2011). Effects of Age on Concurrent Vowel Perception in Acoustic and Simulated Electroacoustic Hearing. Journal of Speech, Language, and Hearing Research, 54(1), 190-210. DOI:10.1044/1092-4388(2010/09-0145)

Assmann, P. F., \& Summerfield, Q. (1990). Modeling the perception of concurrent vowels: Vowels with different fundamental frequencies. Journal of the Acoustical Society of America, 88(2), 680-697.

Baer, T., \& Moore, B. C. J. (1993). Effects of spectral smearing on the intelligibility of sentences in noise. Journal of the Acoustical Society of America, 94(3), 1229-1241.

Baer, T., \& Moore, B. C. J. (1994). Effects of spectral smearing on the intelligibility of sentences in the presence of interfering speech. Journal of the Acoustical Society of America, 95(4), 2277-2280.

Bolia, R. S., Nelson, W. T., Ericson, M. A., \& Simpson, B. D. (2000). A speech corpus for multitalker communications research. Journal of the Acoustical Society of America, 107(2), 1065-1066.

Brown, C. A., \& Bacon, S. P. (2009). Low-frequency speech cues and simulated electricacoustic hearing. Journal of the Acoustical Society of America, 125(3), 1658-1665. DOI:10.1121/1.3068441 
Brown, C. A., \& Bacon, S. P. (2010). Fundamental frequency and speech intelligibility in background noise. Hearing Research, 266, 52-59. DOI:10.1016/j.heares.2009.08.001

Chang, J. E., Bai, J. Y., \& Zeng, F.-G. (2006). Unintelligible Low-Frequency Sound Enhances Simulated Cochlear-Implant Speech Recognition in Noise. IEEE Transactions on Biomedical Engineering, 53(12), 2598-2601. DOI: 10.1109/TBME.2006.883793

Clark, G. A. (2003). Cochlear Implants: Fundamentals and applications. New York: Springer.

Clark, B. G., Franz, B. K.-H. G., Pyman, B. C., \& Webb, R. L. (1991). Surgery for multichannel cochlear implantation. In H. Cooper (Ed.), Cochlear Implants: A Practical Guide (pp. 169-200). London: Whurr Publishers Ltd.

Cullington, H. E., \& Zeng, F.-G. (2008). Speech recognition with varying numbers and types of competing talkers by normal-hearing, cochlear-implant, and implant simulation subjects. Journal of the Acoustical Society of America, 123(1), 450-461. DOI:10.1121/1.2805617

Dorman, M. F., Loizou, P. C., \& Rainey, D. (1997). Speech intelligibility as a function of the number of channels of stimulation for signal processors using sine-wave and noiseband outputs. Journal of the Acoustical Society of America, 102(4), 2403-2411.

Dorman, M. F., Spahr, A. J., Loizou, P. C., Dana, C. J., \& Schmidt, J. S. (2005). Acoustic Simulation of Combined Electric and Acoustic Hearing (EAS). Ear \& Hearing, 26(4), 371-380.

Dorman, M. F., Gifford, R. H., Spahr, A. J., \& McKarns, S. A. (2008). The Benefits of Combining Acoustic and Electric Stimulation for the Recognition of Speech, Voice and Melodies. Audiology \& Neurotology, 13, 105-112. DOI:10.1159/000111782

Dudley, H. (1939). Remaking Speech. Journal of the Acoustical Society of America, 11(2), 169-177. 
Eddins, D. A., \& Liu, C. (2012). Psychometric properties of the coordinate response measure corpus with various types of background interference. Journal of the Acoustical Society of America, 131(2), EL177-EL183. DOI: 10.1121/1.3678680

Fishman, K. E., Shannon, R. V., \& Slattery, W. H. (1997). Speech Recognition as a Function of the Number of Electrodes Used in the SPEAK Cochlear Implant Speech Processor. Journal of Speech, Language, and Hearing Research, 40(5), 1201-1215.

Friesen, L., Shannon, R., Baskent, D., \& Wang, X. (2001). Speech recognition in noise as a function of the number of spectral channels: Comparison of acoustic hearing and cochlear implants. Journal of the Acoustical Society of America, 110(2), 1150-1163. DOI:10.1121/1.1381538

Gantz, B. J., Turner, C., Gfeller, K. E., \& Lowder, M. W. (2005). Preservation of Hearing in Cochlear Implant Surgery: Advantages of Combined Electrical and Acoustical Speech Processing. The Laryngoscope, 115, 796-802. DOI:10.1097/01.MLG.0000157695.07536.D2

Gantz, B. J., Turner, C., Gfeller, K. E. (2006). Acoustic plus Electric Speech Processing: Preliminary Results of a Multicenter Clinical Trial of the Iowa/Nucleus Hybrid Implant. Audiology \& Neurotology, 11(1), 63-68. DOI:10.1159/000095616

Gfeller, K., Turner, C., Mehr, M., Woodworth, G., Fearn, R., Knutson, J. F., Witt, S., \& Stordahl, J. (2002). Recognition of familiar melodies by adult cochlear implant recipients and normal-hearing adults. Cochlear Implant International, 3(1), 29-53.

Glasberg, B. R., \& Moore, B. C. J. (1986). Auditory filter shapes in subjects with unilateral and bilateral cochlear impairments. Journal of the Acoustical Society of America, 79(4), 1020-1033.

Glasberg, B. R., \& Moore, B. C. J. (1990). Derivation of auditory filter shapes from notchednoise data. Hearing Research, 47, 103-138. 
Golub, J. S., Won, J. H., Drennan, W. R., Worman, T. D., \& Rubinstein, J. T. (2012). Spectral and Temporal Measures in Hybrid Cochlear Implant Users: On the Mechanism of Electroacoustic Hearing Benefits. Otology \& Neurology, 33(2), 147153.

Gstoettner, W., Helbig, S., Settevendemie, C., Baumann, U., Wagenblast, J., \& Arnoldner, C. (2009). A new electrode for residual hearing preservation in cochlear implantation: first clinical results. Acta Oto-Laryngologica, 129, 372-379.

DOI: $10.1080 / 00016480802552568$

Helbig, S., Baumann, U., Helbig, M., von Malsen-Waldkirch, N., \& Gstoettner, W. (2008). A New Combined Speech Processor for Electric and Acoustic Stimulation - Eight Months Experience. Journal for Oto-Rhino-Laryngology, Head and Neck Surgery, 70, 359-365. DOI:10.1159/000163031

Kiefer, J., Pok, M., Adunka, O., Stürzebecher, E., Baumgartner, W., Schmidt, M., Tillein, J., Ye, Q., \& Gstoettner, W. (2005). Combined Electric and Acoustic Stimulation of the Auditory System: Results of a Clinical Study. Audiology \& Neurotology, 10, 134-144. DOI:10.1159/000084023

Kiefer, J., Gstoettner, W., Baumgartner, W., Pok, S. M., Tillein, J., Ye, Q., \& Von Ilberg, C. (2004). Conservation of Low-frequency Hearing in Cochlear Implantation. Acta OtoLaryngologica, 124, 272-280. DOI:10.1080/00016480310000755

Kong, Y.-Y., Carlyon, R. P., (2007). Improved speech recognition in noise in simulated binaurally combined acoustic and electric stimulation. Journal of the Acoustical Society of America, 121(6), 3717-3727. DOI:10.1121/1.2717408

Kong, Y.-Y., Stickney, G. S., Zeng, F.-G. (2005). Speech and melody recognition in binaurally combined acoustic and electric hearing. Journal of the Acoustical Society of America, 117(3), 1351-1361. DOI:10.1121/1.1857526 
Li, N., \& Loizou, P. C. (2008). A glimpsing account for the benefit of simulated combined acoustic and electric hearing. Journal of the Acoustical Society of America, 123(4), 2287-2294. DOI:10.1121/1.2839013

Li, N., \& Loizou, P. C. (2009). Factors affecting masking release in cochlear-implant vocoded speech. Journal of the Acoustical Society of America, 126(1), 338-346. DOI:10.1121/1.3133702

Lorens, A., Polak, M., Piotrowska, A., \& Skarzynski, H. (2008). Outcomes of Treatment of Partial Deafness With Cochlear Implantation: A DUET Study. The Laryngoscope, $118,288-294$.

Luetje, C. M., Thedinger, B. S., Buckler, L. R., Dawson, K. L., \& Lisbona, K. L. (2007). Hybrid Cochlear Implantation: Clinical Results and Critical Review in 13 Cases. Otology \& Neurology, 28(4), 473-478.

Moore, B. C. J., Glasberg, B. R., \& Simpson, A. (1992). Evaluation of a method of simulating reduced frequency selectivity. Journal of the Acoustical Society of America, 91(6), 3402-3423.

Moore, B. C. J. (2013). An Introduction to the Psychology of Hearing, $6^{\text {th }}$ Ed. Leiden, Brill.

Patterson, R. D. (1976). Auditory filter shapes derived with noise stimuli. Journal of the Acoustical Society of America,59(3), 640-654.

Patterson, R. D., Nimmo-Smith, I., Weber, D. L., \& Milroy, R. (1982). The deterioration of hearing with age: frequency selectivity, the critical ratio, the audiogram, and speech threshold. Journal of the Acoustical Society of America, 72(6), 1788-1803.

Qin, M. K., \& Oxenham, A. J. (2005). Effects of Envelope-Vocoder Processing on F0 Discrimination and Concurrent-Vowel Identification. Ear and Hearing, 26, 451-460. 
Qin, M. K., \& Oxenham, A. J. (2006). Effects of introducing unprocessed low-frequency information on the reception of envelope-vocoder processed speech. Journal of the Acoustical Society of America, 119(4), 2417-2426. DOI: 10.1121/1.2178719

Sampaio, A., Araújo, M., \& Oliveira, C. (2011). New Criteria of Indication and Selection of Patients to Cochlear Implant. International Journal of Otolaryngology, 2011, 1-13. DOI:10.1155/2011/573968

Seldran, F., Thai-Van, H., Truy, E., Berger-Vachon, C., Collet, L., \& Gallego, S. (2010). Simulation of an EAS Implant with a Hybrid Vocoder. Cochlear Implants International, 11(1), 125-129. DOI:10.1179/146701010X12671177544302

Shannon, R. V., Fu, Q., Galvin, J., \& Friesen, L. (2004). Speech perception with cochlear implants. In F. Zeng, A. N. Popper, \& R. R. Fay (Eds.), Cochlear implants: Auditory prostheses and electric hearing (pp. 334-376). New York: Springer.

Shannon, R. V., Zeng, F., Kamath, V., Wygonski, J., \& Ekelid, M. (1995). Speech Recognition with Primarily Temporal Cues. Science, 270, 303-304.

Sheffield, B. M., \& Zeng, F.-G. (2012). The relative phonetic contributions of a cochlear implant and residual acoustic hearing to bimodal speech perception. Journal of the Acoustical Society of America, 131(1), 518-530. DOI:10.1121/1.3662074

Stickney, G. S., Assmann, P. F., Chang, J., \& Zeng, F.-G. (2007). Effects of cochlear implant processing and fundamental frequency on the intelligibility of competing sentences. Journal of the Acoustical Society of America, 122(2), 1069-1078. DOI:10.1121/1.2750159

Turner, C. W. (2006). Hearing Loss and the Limits of Amplification. Audiology \& Neurotology, 11(1), 2-5. DOI:10.1159/000095606 
Turner, C. W., Gantz, B. J., Vidal, C., Behrens, A., \& Henry, B. A. (2004). Speech recognition in noise for cochlear implant listeners: Benefits of residual acoustic hearing. Journal of the Acoustical Society of America, 115(4), 1729-1735. DOI:10.1121/1.1687425

Turner, C., Gantz, B. J., \& Reiss, L. (2008). Integration of acoustic and electrical hearing. Journal of Rehabilitation Research \& Development, 45(5), 769-778. DOI:10.1682/JRRD.2007.05.0065

Turner, C. W., Gantz, B. J., Karsten, S., Fowler, J., \& Reiss, L. A. (2010). Impact of Hair Cell Preservation in Cochlear Implantation: Combined Electric and Acoustic Hearing. Otology \& Neurology, 31(8), 1227-1232.

Vickers, D. A., Moore, B. C. J., \& Baer, T. (2001). Effects of low-pass filtering on the intelligibility of speech in quiet for people with and without dead regions at high frequencies. Journal of the Acoustical Society of America, 110(2), 1164-1175. DOI:10.1121/1.1381534

Von Békésy, G. (1960). Experiments in Hearing. Michigan, US: McGraw.

Von Ilberg, C. A., Baumann, U., Kiefer, J., Tillein, J., \& Adunka, O. F. (2011). ElectricAcoustic Stimulation of the Auditory System: A Review of the First Decade. Audiology \& Neurotology, 16(2), 1-30. DOI:10.1159/000327765

Von Ilberg, C. A., Kiefer, J., Tillein, J., Pfenningdorff, T., Hartmann, R., Stürzebecher, E., \& Klinke, R. (1999). Electric-Acoustic Stimulation of the Auditory System: New Technology for Severe Hearing Loss. Journal for Oto-Rhino-Laryngology and its Related Specialities, 61, 334-340.

Whitmal III, N. A., Poissant, S. F., Freyman, R. L., \& Helfer, K. S. (2007). Speech intelligibility in cochlear implant simulations: Effects of carrier type, interfering noise, and subject experience. Journal of the Acoustical Society of America, 122(4), 2376-2388. DOI:10.1121/1.2773993 
Wilson, B. S. (2004). Engineering design of cochlear implants. In F. Zeng, A. N. Popper, \& R. R. Fay (Eds.), Cochlear implants: Auditory prostheses and electric hearing (pp. 14-52). New York: Springer.

Wilson, B. S., Finley, C. C., Lawson, D. T., Wolford, R. D., Eddington, D. K., \& Rabinowitz, W. M. (1991). Better speech recognition with cochlear implants. Nature, 352(6332), 236-238.

Wilson, B. S., Lawson, D. T., Müller, J. M., Tyler, R. S., \& Kiefer, J. (2003). Cochlear Implants: Some Likely Next Steps. Annual Review of Biomedical Engineering, 5, 207-249. DOI:10.1146/annurev.bioeng.5.040202.121645

Yang, H.-I., Zeng, F.-G. (2013). Reduced acoustic and electric integration in concurrentVowel recognition. Scientific Reports, 3, 1419. DOI:10.1038/srep01419 
APPENDIX A - Information Sheet

University of Canterbury

Department of Communication Disorders

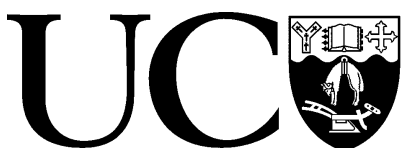

Private Bag 4800

Christchurch 8140

New Zealand

\section{The Effect of Spectral Smearing and Elevated Thresholds on Speech in Noise Recognition in Simulated Electric-Acoustic Hearing}

\section{Information Sheet}

You are invited to participate in this project carried out by Aretha Mulder, Master of Audiology student from the Department of Communication Disorders at the University of Canterbury. The aim of this project is to investigate the effects of the degree of low frequency hearing on speech in background noise in simulated cochlear implant listening.

Your involvement in this project will involve one session lasting approximately 2 hours, including rest breaks as needed. As participants are required to have normal hearing, you will be asked to undergo a brief hearing screen prior to testing. Following the hearing screen you will be required to listen to sentences in the presence of background noise presented via headphones and to identify two key words from each sentence by clicking on response boxes on a computer screen. Sentences will be manipulated in a variety of ways to simulate different conditions and will vary in their level of difficulty. As reimbursement for your participation in this project you will be offered a $\$ 30$ grocery voucher.

In the performance of the tasks and application of the procedures there is the risk that you may be identified as having a hearing loss, which may be distressing. If the hearing screen identifies any participant as having a hearing loss, participants will be referred to the University of Canterbury Speech and Hearing clinic where they will be offered a full diagnostic assessment and ongoing support.

You may receive a copy of the project results by contacting the researcher at the conclusion of the project. Participation is voluntary and you have the right to withdraw at any stage without penalty. If you withdraw, I will remove information relating to you prior to November 2013 at which stage removal of data will become impossible.

The results of the project may be published, and a Master's Thesis is a public document, accessible via the University of Canterbury library database but you may be assured of the complete confidentiality of data gathered in this investigation: the identity of participants will not be made public. To ensure anonymity and confidentiality, the information gathered will be assigned a number and all identifiable information removed. Data and back-up files will 
be kept on hard drives which are accessible only to the investigators. This data will be kept for five years after which time it will be destroyed.

The project is being carried out as a requirement for a Masters of Audiology by Aretha Mulder under the supervision of Donal Sinex, who can be contacted at donal.sinex@canterbury.ac.nz. He will be pleased to discuss any concerns you may have about participation in the project.

This project has been reviewed and approved by the University of Canterbury Human Ethics Committee, and participants should address any complaints to The Chair, Human Ethics Committee, University of Canterbury, Private Bag 4800, Christchurch (humanethics@canterbury.ac.nz).

If you agree to participate in the study, you are asked to complete the consent form. Aretha Mulder 
APPENDIX B - Consent Form

University of Canterbury

Department of Communication Disorders

Private Bag 4800

Te Whare Wānanga o Waitaha CHRISTCHURCH NEW ZEALAND

Christchurch 8140

New Zealand

\section{The Effect of Spectral Smearing and Elevated Thresholds on Speech in Noise Recognition in Simulated Electric-Acoustic Hearing}

\section{Consent Form}

I have been given a full explanation of this project and have had the opportunity to ask questions.

I understand what is required of me if I agree to take part in the research.

I understand that participation is voluntary and I may withdraw at any time without penalty.

Withdrawal of participation will also include the withdrawal of any information I have provided should this remain practically achievable.

I understand that any information or opinions I provide will be kept confidential to the researcher and that any published or reported results will not identify the participants. I understand that a thesis is a public document and will be available through the UC Library.

I understand that all data collected for the study will be kept in locked and secure facilities and/or in password protected electronic form and will be destroyed after five years.

I understand the risks associated with taking part and how they will be managed.

I understand that I am able to receive a report on the findings of the study by contacting the researcher at the conclusion of the project.

I understand that I can contact the researcher, Aretha Mulder at mobile: 0273450759 or email: aretha.mulder@pg.canterbury.ac.nz or supervisor, Donal Sinex at email:

donal.sinex@canterbury.ac.nz for further information. If I have any complaints, I can contact the Chair of the University of Canterbury Human Ethics Committee, Private Bag 4800, Christchurch (human-ethics@ canterbury.ac.nz)

By signing below, I agree to participate in this research project.

Name:

Signature:

Date: 